Supporting Information for:

\title{
Planar Chiral Cyclic Amine and Its Derivatives: Synthesis and Stereochemical Behavior
}

Katsuhiko Tomooka,* Masaki Suzuki, Maki Shimada, Syun-ichi Yanagitsuru, and Kazuhiro Uehara

\section{General Experimental.}

All reactions were carried out with dry glassware under an atmosphere of argon otherwise noted. Dry THF, $\mathrm{Et}_{2} \mathrm{O}$ and $\mathrm{CH}_{2} \mathrm{Cl}_{2}$ were purchased from Kanto Chemical Co., Inc and used without purification. ${ }^{1} \mathrm{H}$ NMR spectra were recorded on a Varian Gemine-2000 (300 MHz) or a Varian Mercury (300 MHz) using $\mathrm{CDCl}_{3}$ as solvent: $\mathrm{CHCl}_{3}\left({ }^{1} \mathrm{H}, \delta\right.$ 7.26) was used as an internal reference. Peak multiplicities were given as followed: s, singlet; $d$, doublet; $\mathrm{t}$, triplet; $\mathrm{m}$, multiplet; br, broad. ${ }^{13} \mathrm{C}$ NMR spectra were recorded on a Varian Gemine-2000 (75 MHz) or a Varian Mercury (75 MHz) using $\mathrm{CDCl}_{3}$ as solvent: $\mathrm{CDCl}_{3}\left({ }^{13} \mathrm{C}, \delta 77.1\right)$ was used as an internal reference. Optical rotations were measured on a digital polarimeter (JASCO DIP-370). Infrared spectra were recorded on a Fourier transfer infrared spectrophotometer (Perkin Elmer SpectrumOne) as neat liquid on $\mathrm{NaCl}$ plates and as crystal used diffuse reflector. High performance liquid chromatography (HPLC) was performed on a SHIMADZU SPD-6A equipped with a SHIMADZU LC-9A or a JASCO CD-2095 equipped with a JASCO PU-2089 using Daisel CHIRALCEL OD-H column (0.46 cm x $25 \mathrm{~cm}),(2.00 \mathrm{~cm} \times 25 \mathrm{~cm})$ or OD-RH column $(0.46 \mathrm{~cm} \times 15 \mathrm{~cm})$. CD spectra were measured on a JASCO CD-2095. Thin-layer chromatography (TLC) was carried out on silica gel $60 \mathrm{~F}_{254}$ (Merck 5715) plates. Developed plates were visualized by ultraviolet illumination at $254 \mathrm{~nm}$ and by heating on a hot plate after staining with a $4 \%$ solution of phosphomolybdic acid in ethanol, a $2.5 \%$ solution of $p$-anisaldehyde in ethanol, or an aqeous solution of potassium permanganate. Column chromatography was performed using Kanto 60N (63-210 $\mu \mathrm{m})$. Elemental analyses were performed by the Analytical Facility at the Research Laboratory of Resources Utilization, Tokyo Institute of Technology.

acetic acid (2Z,6E)-8-hydroxy-3,7-dimethyl-octa-2,6-dienylester (2)

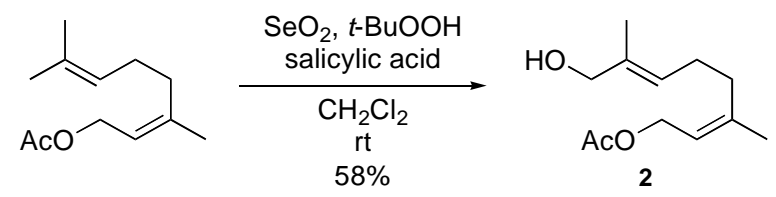

This compound was prepared by a slightly modified procedure of the one described by: Marshall, J. A.; Lebreton, J. J. Org. Chem. 1988, 53, 4108-4112.

To a solution of a catalytic amount of $\mathrm{SeO}_{2}(79.6 \mathrm{mg}, 0.695 \mathrm{mmol})$ and salicylic acid $(96.0 \mathrm{mg}, 0.695$ mmol) in dry $\mathrm{CH}_{2} \mathrm{Cl}_{2}(10 \mathrm{~mL})$ at room temperature was added anhydrous TBHP in $\mathrm{CH}_{2} \mathrm{Cl}_{2}(30 \mathrm{~mL})$, which was prepared from TBHP in water $(3.5 \mathrm{~mL}$ of $70 \mathrm{wt} \%$ solution, $25.0 \mathrm{mmol}$ ) by azeotropic distillation of water and $\mathrm{CH}_{2} \mathrm{Cl}_{2}$. Neryl acetate $(1.5 \mathrm{~mL}, 6.95 \mathrm{mmol})$ was added to the mixture, then the resulting mixture was stirred at that temperature for $23 \mathrm{~h}$. The reaction was quenched with aq satd $\mathrm{Na}_{2} \mathrm{~S}_{2} \mathrm{O}_{3}$ and extracted with $\mathrm{CH}_{2} \mathrm{Cl}_{2}$. The combined organic phase was dried over $\mathrm{Na}_{2} \mathrm{SO}_{4}$, filtered and the solvent was removed in 
vacuo. The residue was purified by silica gel chromatography, affording $234 \mathrm{mg}(17 \%)$ of the recovered starting material (hexane/AcOEt $=10 / 1), 224 \mathrm{mg}(15 \%)$ of aldehyde (hexane/AcOEt $=6 / 1)$ and $861 \mathrm{mg}$ $(58 \%)$ of alcohol 2 (hexane/AcOEt $=3 / 1$ to $1 / 1) .{ }^{1} \mathrm{H}$ NMR $\left(300 \mathrm{MHz}, \mathrm{CDCl}_{3}\right): \delta 5.39$ (brs, $\left.1 \mathrm{H}\right), 5.36$ (t, $J=$ $7.2 \mathrm{~Hz}, 1 \mathrm{H}), 4.56$ (d, J=7.2 Hz, 2H), 3.99 (s, 2H), 2.16 (s, 2H), 2.15 (s, 2H), 2.05 (s, 3H), 1.77 (s, 3H), $1.66(\mathrm{~s}, 3 \mathrm{H}) ;{ }^{13} \mathrm{C}$ NMR $\left(75 \mathrm{MHz}, \mathrm{CDCl}_{3}\right): \delta 171.2,142.0,135.7,125.0,119.7,68.9,61.3,31.8,26.0,23.5$, 21.2, 13.8; IR (neat, $\mathrm{cm}^{-1}$ ): 3432, 2937, 2862, 1739, 1670, 1445, 1379, 1236, 1022, 954, 852, 743.

\section{methyl- $N$-( $p$-toluenesulfonyl)carbamate}

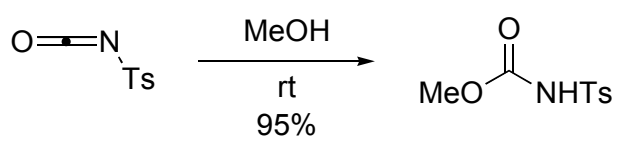

This compound was prepared based on the literature procedure: Henry, J. R.; Marcin, L. R.; McIntosh, M. C.; Scola, P. M.; Harris, G. D., Jr.; Weinreb, S. M. Tetrahedron Lett. 1989, 30, 5709-5712.

$p$-Toluenesulfonyl isocyanate $(25.0 \mathrm{~g}, 19.4 \mathrm{~mL}, 0.127 \mathrm{~mol})$ was added to dry $\mathrm{MeOH}(50 \mathrm{~mL})$ at $0{ }^{\circ} \mathrm{C}$ dropwise. Then the temperature was allowed to rise to room temperature. The mixture was stirred for $3 \mathrm{~h}$, concentrated in vacuo and the crude product was recrystallized from hexane $/ \mathrm{CH}_{2} \mathrm{Cl}_{2}$ to afford $27.5 \mathrm{~g}(95 \%)$ of $N$-methoxy- $N$-tosylcarbamate as a white solid. ${ }^{1} \mathrm{H}$ NMR (300 MHz, $\left.\mathrm{CDCl}_{3}\right): \delta 7.93(\mathrm{~d}, J=8.4 \mathrm{~Hz}, 2 \mathrm{H})$, 7.50-7.45 (brs, $1 \mathrm{H}), 7.34$ (d, $J=8.4 \mathrm{~Hz}, 2 \mathrm{H}), 3.70(\mathrm{~s}, 3 \mathrm{H}), 2.45(\mathrm{~s}, 3 \mathrm{H}) ;{ }^{13} \mathrm{C}$ NMR $\left(75 \mathrm{MHz}, \mathrm{CDCl}_{3}\right): \delta$ 150.9, 145.1, 135.3, 129.6, 128.3, 53.7, 21.9; IR (neat, $\mathrm{cm}^{-1}$ ): 3244, 1756, 1595, 1455, 1353, 1229, 1091, $872,771,734,663$.

\section{$N$-methoxy-carbonyl- $N$-((2Z,6E)-8-acetyl-2,6-dimethyl-octa-2,6-dienyl)-p-toluenesulfonamide (3)}

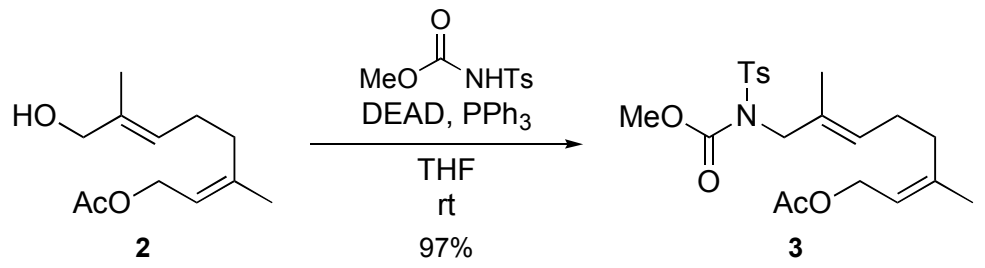

To a solution of $2(2.62 \mathrm{~g}, 12.3 \mathrm{mmol})$ in dry THF $(155 \mathrm{~mL})$ at room temperature was added methyl- $N$-( $p$-toluenesulfonyl)carbamate $(3.21 \mathrm{~g}, 14.0 \mathrm{mmol})$, triphenylphosphine $(3.67 \mathrm{~g}, 14.0 \mathrm{mmol})$ and DEAD $(6.35 \mathrm{~mL}$ of $40 \mathrm{wt} \%$ in toluene, $14.0 \mathrm{mmol})$. The mixture was stirred at that temperature for $2.7 \mathrm{~h}$, concentrated in vacuo and the residue was purified by silica gel chromatography (hexane/AcOEt $=30 / 1$ to 1/1) to afford $4.91 \mathrm{~g}$ of 3 (97\%). ${ }^{1} \mathrm{H}$ NMR $\left(300 \mathrm{MHz}, \mathrm{CDCl}_{3}\right): \delta 7.81$ (d, $\left.J=8.4 \mathrm{~Hz}, 2 \mathrm{H}\right), 7.29$ (d, $J=8.4$ Hz, 2H), 5.37 (t, $J=7.2 \mathrm{~Hz}, 1 \mathrm{H}), 5.34$ (s, 1H), 4.55 (d, $J=7.2 \mathrm{~Hz}, 2 \mathrm{H}), 4.37$ (s, 2H), 3.68 (s, 3H), 2.43 (s, $3 \mathrm{H}), 2.15(\mathrm{~s}, 2 \mathrm{H}), 2.14(\mathrm{~s}, 2 \mathrm{H}), 2.04(\mathrm{~s}, 3 \mathrm{H}), 1.77(\mathrm{~s}, 3 \mathrm{H}), 1.60(\mathrm{~s}, 3 \mathrm{H}) ;{ }^{13} \mathrm{C}$ NMR $\left(75 \mathrm{MHz}, \mathrm{CDCl}_{3}\right): \delta$ 170.9, 152.9, 144.5, 142.0, 136.4, 130.6, 129.2, 128.5, 126.5, 119.4, 61.1, 53.5, 51.8, 31.8, 26.6, 23.6, 21.8, 21.2, 14.3; IR (neat, $\mathrm{cm}^{-1}$ ): 2958, 1738, 1597, 1445, 1360, 1171, 1021, 956, 771, 652; Anal. Calcd. for $\mathrm{C}_{21} \mathrm{H}_{29} \mathrm{NO}_{6} \mathrm{~S}: \mathrm{C}, 59.56 ; \mathrm{H}, 6.90 ; \mathrm{N}, 3.31 ; \mathrm{S}, 7.57$. Found: C, 59.43; H, 7.00 N, 3.24; S, 7.39. 
$N$-((2E,6Z)-8-hydroxy-2,6-dimethyl-octa-2,6-dienyl)-p-toluenesulfonamide (4)

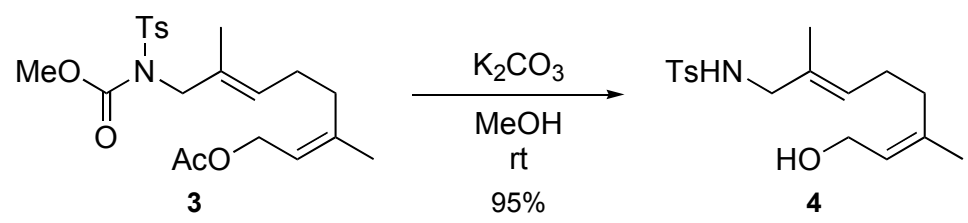

To a solution of $3(8.47 \mathrm{~g}, 20.0 \mathrm{mmol})$ in $\mathrm{MeOH}(200 \mathrm{~mL})$ at room temperature was added $\mathrm{K}_{2} \mathrm{CO}_{3}(3.59$ $\mathrm{g}, 26.0 \mathrm{mmol})$. After the mixture was stirred for $20 \mathrm{~h}$ at that temperature, the reaction was quenched with aq satd $\mathrm{NH}_{4} \mathrm{Cl}$, then extracted with AcOEt. The combined organic phase was dried over $\mathrm{Na}_{2} \mathrm{SO}_{4}$, filtered and the solvent was removed in vacuo. The crude product was purified by silica gel chromatography (hexane/AcOEt $=20 / 1$ to $1 / 1)$ to afford $6.04 \mathrm{~g}$ of $4(95 \%) .{ }^{1} \mathrm{H}$ NMR $\left(300 \mathrm{MHz}, \mathrm{CDCl}_{3}\right): \delta 7.73(\mathrm{~d}, J=8.1$ $\mathrm{Hz}, 2 \mathrm{H}), 7.27(\mathrm{~d}, J=8.1 \mathrm{~Hz}, 2 \mathrm{H}), 5.52(\mathrm{br}, 1 \mathrm{H}), 5.39(\mathrm{t}, J=6.6 \mathrm{~Hz}, 1 \mathrm{H}), 5.26(\mathrm{t}, J=6.1 \mathrm{~Hz}, 1 \mathrm{H}), 4.05(\mathrm{~d}, J$ $=6.6 \mathrm{~Hz}, 2 \mathrm{H}), 3.38(\mathrm{~d}, J=6.1 \mathrm{~Hz}, 2 \mathrm{H}), 2.42(\mathrm{~s}, 3 \mathrm{H}), 2.07(\mathrm{~s}, 2 \mathrm{H}), 2.06(\mathrm{~s}, 2 \mathrm{H}), 1.71(\mathrm{~s}, 3 \mathrm{H}), 1.57(\mathrm{~s}, 3 \mathrm{H})$; ${ }^{13} \mathrm{C}$ NMR $\left(75 \mathrm{MHz}, \mathrm{CDCl}_{3}\right): \delta 143.0,138.3,137.1,131.3,129.6,127.2,127.0,124.7,59.0,51.0,31.2,25.5$, 23.4, 21.7, 14.7; IR (neat, $\mathrm{cm}^{-1}$ ): 3501, 3289, 2921, 1668, 1599, 1446, 1324, 1157, 1094, 995, 815, 666; Anal. Calcd. for $\mathrm{C}_{17} \mathrm{H}_{25} \mathrm{NO}_{3} \mathrm{~S}$ : C, 63.13; H, 7.79; N, 4.33; S, 9.91. Found: C, 62.62; H, 7.92; N, 4.27; S, 9.71 .

(3E,7Z)-3,7-dimethyl-1-(p-toluenesulfonyl)-2,5,6,9-tetrahydro-1 $H$-azonine (1a)

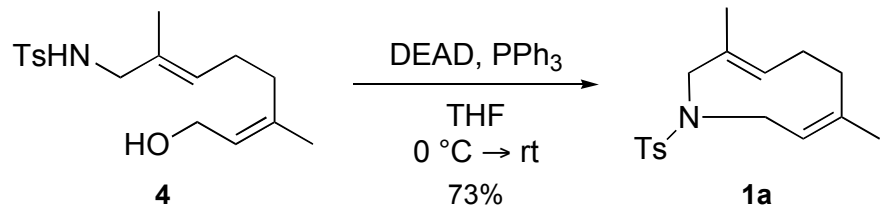

To a solution of $4(1.12 \mathrm{~g}, 3.48 \mathrm{mmol})$ and triphenylphosphine $(1.47 \mathrm{~g}, 5.59 \mathrm{mmol})$ in dry THF $(500 \mathrm{~mL})$ at $0{ }^{\circ} \mathrm{C}$ was added DEAD $(2.53 \mathrm{~mL}$, of $40 \mathrm{wt} \%$ in toluene, $5.58 \mathrm{mmol})$. Then the temperature was allowed to rise to room temperature. The mixture was stirred for $47 \mathrm{~h}$, concentrated in vacuo and the residue was purified by silica gel chromatography (hexane/AcOEt $=20 / 1$ to $5 / 1$ ) to afford $776 \mathrm{mg}$ of 1 a $(73 \%)$ as a white solid and a trace amount of dimerized product $\left(<1 \%\right.$, analyzed by ${ }^{1} \mathrm{H}$ NMR). ${ }^{1} \mathrm{H}$ NMR $(300 \mathrm{MHz}$, $\left.\mathrm{CDCl}_{3}\right): \delta 7.67(\mathrm{~d}, J=8.1 \mathrm{~Hz}, 2 \mathrm{H}), 7.30(\mathrm{~d}, J=8.1 \mathrm{~Hz}, 2 \mathrm{H}), 5.33-5.30(\mathrm{~m}, 1 \mathrm{H}), 5.28-5.26(\mathrm{~m}, 1 \mathrm{H}), 4.25(\mathrm{~d}$, $J=10.2 \mathrm{~Hz}, 1 \mathrm{H}), 3.86(\mathrm{dd}, J=14.1,11.7 \mathrm{~Hz}, 1 \mathrm{H}), 3.01(\mathrm{dd}, J=14.1,4.2 \mathrm{~Hz}, 1 \mathrm{H}), 3.00(\mathrm{~d}, J=10.2 \mathrm{~Hz}$, $1 \mathrm{H}), 2.43$ (s, 3H), 2.06-2.00 (m, 2H), 1.94-1.90 (m, 2H), 1.73 (s, 3H), 1.54 (s, 3H); ${ }^{13} \mathrm{C}$ NMR (75 MHz, $\left.\mathrm{CDCl}_{3}\right): \delta 142.9,136.2,134.3,134.2,133.4,129.6,126.9,126.1,59.0,45.1,32.0,25.7,25.4,21.6,16.6$; IR (neat, $\mathrm{cm}^{-1}$ ): 2944, 2463, 1918, 1808, 1654, 1596, 1332, 1210, 811, 735, 657, 594, 526; Analytical HPLC (column: CHIRALCEL OD-H $(0.46 \mathrm{~cm} \times 25 \mathrm{~cm})$, eluent: hexane/EtOH $=1 / 1$, flow rate: $0.5 \mathrm{~mL} / \mathrm{min}$, detection: UV $254 \mathrm{~nm}$, temperature: $\mathrm{rt}) t_{R}=9.9 \mathrm{~min}$ for $(R)$-isomer, $13.1 \mathrm{~min}$ for $(S)$-isomer; Preparative HPLC (column: CHIRALCEL OD-H $(2.00 \mathrm{~cm} \times 25 \mathrm{~cm}$ ), eluent: hexane/EtOH $=1 / 1$, flow rate: 3.0 $\mathrm{mL} / \mathrm{min}$, detection: UV $254 \mathrm{~nm}$, temperature: $\mathrm{rt}) t_{R}=30.2 \mathrm{~min}$ for $(R)$-isomer, $41.0 \mathrm{~min}$ for $(S)$-isomer; Optical rotation value: $[\alpha]_{\mathrm{D}}{ }^{27}=-93.3\left(c=0.95, \mathrm{CHCl}_{3}\right)$ for $(R)$-isomer $(>98 \% \mathrm{ee}),[\alpha]_{\mathrm{D}}{ }^{28}=+92.1(c=0.49$, $\mathrm{CHCl}_{3}$ ) for $(S)$-isomer $\left(>98 \%\right.$ ee); Anal. Calcd. for $\mathrm{C}_{17} \mathrm{H}_{23} \mathrm{NO}_{2} \mathrm{~S}: \mathrm{C}, 66.85 ; \mathrm{H}, 7.59 ; \mathrm{N}, 4.59 ; \mathrm{S}, 10.50$. Found: C, 62.86; H, 7.48; N, 4.67; S, 10.63 . 
(3E,7Z)-3,7-dimethyl-2,5,6,9-tetrahydro-1 $H$-azonine (1b)

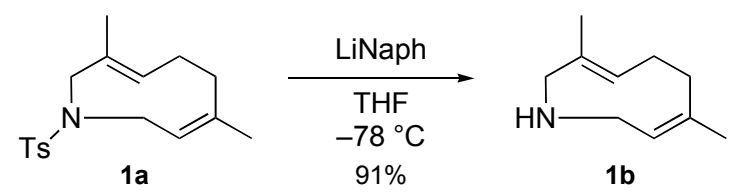

To a solution of $1 \mathbf{a}(104 \mathrm{mg}, 0.340 \mathrm{mmol})$ in $\operatorname{dry} \mathrm{THF}(5 \mathrm{~mL})$ at $-78{ }^{\circ} \mathrm{C}$ was added lithium naphthalenide (1.07 $\mathrm{mL}$ of ca. $0.80 \mathrm{M}$ in THF, $0.850 \mathrm{mmol}$ ). The resulting mixture was stirred for $10 \mathrm{~min}$ at that temperature. Then the reaction was quenched with $1 \mathrm{~N} \mathrm{HCl}(\mathrm{aq})$ and washed with $\mathrm{Et}_{2} \mathrm{O}$ at $\mathrm{pH}$ 1. To the combined water phase was added $1 \mathrm{~N} \mathrm{NaOH}$ (aq) to $\mathrm{pH}$ 13. The solution was extracted with $\mathrm{Et}_{2} \mathrm{O}$, the combined organic phase was dried over $\mathrm{Na}_{2} \mathrm{SO}_{4}$, filtered and the solvent was removed in vacuo to afford $46.6 \mathrm{mg}(91 \%)$ of $\mathbf{1 b}$. This crude amine was used in next step without further purification. ${ }^{1} \mathrm{H}$ NMR (300 $\left.\mathrm{MHz}, \mathrm{CDCl}_{3}\right): \delta 5.38(\mathrm{dd}, J=8.1,5.9 \mathrm{~Hz}, 1 \mathrm{H}), 5.18(\mathrm{dd}, J=11.4,4.8 \mathrm{~Hz}, 1 \mathrm{H}), 3.34(\mathrm{~d}, J=11.1 \mathrm{~Hz}, 1 \mathrm{H})$, $3.11(\mathrm{~d}, J=11.1 \mathrm{~Hz}, 1 \mathrm{H}), 3.05(\mathrm{dd}, J=15.1,4.8 \mathrm{~Hz}, 1 \mathrm{H}), 2.90(\mathrm{dd}, J=15.1,11.4 \mathrm{~Hz}, 1 \mathrm{H}), 2.33$ (brs, $1 \mathrm{H})$, 2.06-1.93 (m, 4H), $1.73(\mathrm{~s}, 3 \mathrm{H}), 1.51(\mathrm{~s}, 3 \mathrm{H}) ;{ }^{13} \mathrm{C} \mathrm{NMR}\left(75 \mathrm{MHz}, \mathrm{CDCl}_{3}\right) ; \delta$ 138.0, 131.9, 130.4, 130.0, 58.8, 43.7, 33.7, 25.5, 25.5, 16.5; IR (neat, $\mathrm{cm}^{-1}$ ): 3303, 2963, 2930, 2865, 1610, 1551, 1457, 1437, 1323, 1276, 1240, 1049, 1012, 876, 811, 671; Optical rotation value: $[\alpha]_{\mathrm{D}}^{27}=+4.73(c=1.27$, EtOH $)$ for $(R)$-isomer $\left(>98 \%\right.$ ee), $[\alpha]_{\mathrm{D}}^{27}=-4.89(c=1.08, \mathrm{EtOH})$ for $(S)$-isomer $(>98 \%$ ee).

\section{(S)-(3E,7Z)-3,7-dimethyl -2,5,6,9- tetrahydro-1 $H$-azoninium $(S)$-methoxy-phenyl-acetate (6)}<smiles>CC1=CCC/C(C)=C/CCNC1</smiles>

$(S)-1 \mathbf{b}$<smiles>COC(C(=O)O)c1ccccc1</smiles>

$(S)-5$<smiles>CCOCCOCCOC(=O)C(OC)c1ccccc1</smiles>

$(+)-6$

To a solution of $(S)-1 \mathbf{b}(>98 \%$ ee, $14.8 \mathrm{mg}, 0.0979 \mathrm{mmol})$ in dry $^{\mathrm{Et}} \mathrm{t}_{2} \mathrm{O}(1 \mathrm{~mL})$ at room temperature was added $(S)-(+)$ - $\alpha$-methoxyphenylacetic acid $5(16.2 \mathrm{mg}, 0.0975 \mathrm{mmol})$ dissolved in dry $\mathrm{CH}_{2} \mathrm{Cl}_{2}(0.5 \mathrm{~mL})$. Crystallization from AcOEt/ $\mathrm{CH}_{2} \mathrm{Cl}_{2}$ afforded single crystals that were suitable for X-ray crystal structure analysis. ${ }^{1} \mathrm{H}$ NMR (300 MHz, $\mathrm{CDCl}_{3}$ ): $\delta$ 10.00-9.20 (br, 2H), $7.48(\mathrm{dd}, J=7.8,1.2 \mathrm{~Hz}, 2 \mathrm{H}), 7.33-7.21$ (m, $3 \mathrm{H}), 5.35-5.27(\mathrm{~m}, 2 \mathrm{H}), 4.59(\mathrm{~s}, 1 \mathrm{H}), 3.56(\mathrm{~d}, J=11.4 \mathrm{~Hz}, 1 \mathrm{H}), 3.36(\mathrm{~s}, 3 \mathrm{H}), 3.25(\mathrm{dd}, J=13.5,3.6 \mathrm{~Hz}$, $1 \mathrm{H}), 3.03(\mathrm{~d}, J=11.4 \mathrm{~Hz}, 1 \mathrm{H}), 2.71(\mathrm{dd}, J=13.5,12.3 \mathrm{~Hz}, 1 \mathrm{H}), 2.17-2.09(\mathrm{~m}, 1 \mathrm{H}), 2.05-1.82(\mathrm{~m}, 3 \mathrm{H}), 1.68$ (s, 3H), 1.42 (s, 3H); ${ }^{13} \mathrm{C}$ NMR (75 MHz, $\mathrm{CDCl}_{3}$ ): $\delta$ 177.0, 140.1, 138.0, 135.0, 131.5, 128.2, 127.4, 127.3, 121.5, 85.5, 57.0, 54.9, 42.1, 33.3, 25.9, 25.4, 16.9; M.p.: $142{ }^{\circ} \mathrm{C}$ (decomposed); IR (neat, $\mathrm{cm}^{-1}$ ): 3419, 2969, 1583, 1455, 1386, 1284, 1197, 1169, 1099, 1073, 1029, 992, 954, 939, 915, 891, 878, 857, 818, 782, $732,697,613,526,505,486$; Optical rotation value: $[\alpha]_{\mathrm{D}}{ }^{30}=+63.1\left(c=1.20, \mathrm{CHCl}_{3}\right)$. 


\section{Procedure for fractional crystallization of salt 6 and preparation of $(S)$-1a}

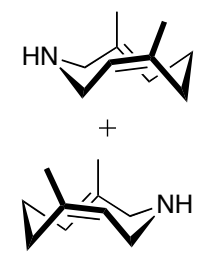

rac-1b

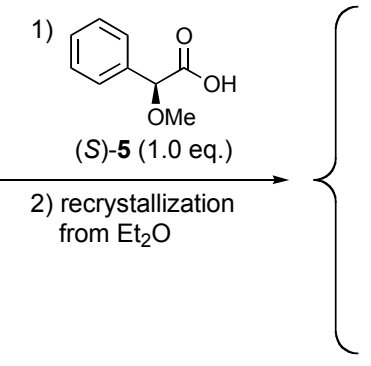

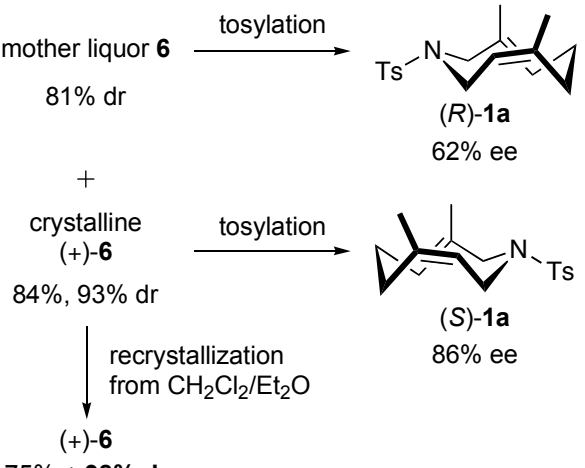

$75 \%,>98 \%$ dr

To a solution of racemic amine $\mathbf{1 b}(143 \mathrm{mg}, 0.943 \mathrm{mmol})$ in $\operatorname{dry}^{\mathrm{Et}} \mathrm{t}_{2} \mathrm{O}(2 \mathrm{~mL})$ at room temperature was added $(S)-(+)-\alpha$-methoxyphenylacetic acid $5(157 \mathrm{mg}, 0.943 \mathrm{mmol})$ dissolved in dry $\mathrm{Et}_{2} \mathrm{O}(3 \mathrm{~mL})$. The solution was recrystallized, then the crystals were collected, washed with dry hexane and dried in vacuo to afford $125 \mathrm{mg}(84 \%)$ of salt (+)-6 as a colorless crystal. Stereochemical purity of the deposited salt (+)-6 and salt 6 in mother liquor were determined as $93 \% \mathrm{dr}, 81 \% \mathrm{dr}$, respectively, by the tosylation $\left(\mathrm{TsCl}_{1} \mathrm{Et}_{3} \mathrm{~N}\right.$, DMAP) followed by the analysis of the resulting $\mathbf{1 a}$ by chiral HPLC.

The stereochemically pure salt $(+)-6(72.9 \mathrm{mg}, 75 \%,>98 \% \mathrm{dr})$ was prepared from the salt $(+)-6(97.5 \mathrm{mg}$, $94 \% \mathrm{dr}$ ) by recrystallization from $\mathrm{CH}_{2} \mathrm{Cl}_{2} / \mathrm{Et}_{2} \mathrm{O}$, twice.

(3E,7Z)-1-Benzyl-3,7-dimethyl-2,5,6,9-tetrahydro-1 $H$-azonine (1c)

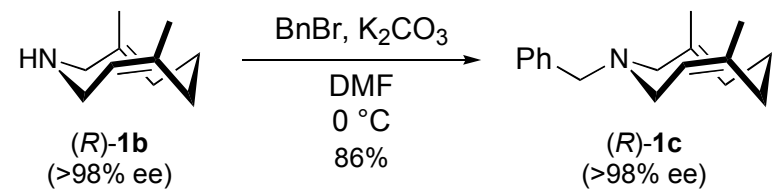

To a solution of $(R)-\mathbf{1 b}(13.9 \mathrm{mg}, 0.0919 \mathrm{mmol},>98 \%$ ee $)$ in dry DMF $(3 \mathrm{~mL})$ at $0{ }^{\circ} \mathrm{C}$ was added benzyl bromide $(11.0 \mu \mathrm{L}, 0.101 \mathrm{mmol})$ and $\mathrm{K}_{2} \mathrm{CO}_{3}(38.1 \mathrm{mg}, 0.276 \mathrm{mmol})$. The resulting mixture was stirred for $10 \mathrm{~h}$ at that temperature. Then the reaction was quenched with aq satd $\mathrm{NaHCO}_{3}$ and extracted with $\mathrm{Et}_{2} \mathrm{O}$. The combined organic phase was washed with brine, dried over $\mathrm{Na}_{2} \mathrm{SO}_{4}$, filtered and the solvent was removed in vacuo. The crude product was purified by silica gel chromatography (hexane/AcOEt $=10 / 1$ to $1 / 1)$ to afford $19.1 \mathrm{mg}$ of $(R)-\mathbf{1 c}\left(86 \%,>98 \%\right.$ ee determined by chiral HPLC analysis). ${ }^{1} \mathrm{H}$ NMR (300 MHz, $\left.\mathrm{CDCl}_{3}\right): \delta 7.41-7.21(\mathrm{~m}, 5 \mathrm{H}), 5.37(\mathrm{dd}, J=8.4,6.3 \mathrm{~Hz}, 1 \mathrm{H}), 5.19(\mathrm{ddd}, J=10.2,5.7,0.9 \mathrm{~Hz}, 1 \mathrm{H}), 3.76(\mathrm{~d}, J$ $=13.8 \mathrm{~Hz}, 1 \mathrm{H}), 3.62(\mathrm{~d}, J=13.8 \mathrm{~Hz}, 1 \mathrm{H}), 3.37(\mathrm{~d}, J=9.3 \mathrm{~Hz}, 1 \mathrm{H}), 2.77(\mathrm{dd}, J=10.5,10.2 \mathrm{~Hz}, 1 \mathrm{H}), 2.73$ (d, $J=9.3 \mathrm{~Hz}, 1 \mathrm{H}), 2.71(\mathrm{dd}, J=10.5,5.7 \mathrm{~Hz}, 1 \mathrm{H}), 2.07-1.91(\mathrm{~m}, 4 \mathrm{H}), 1.70(\mathrm{~s}, 3 \mathrm{H}), 1.57$ (d, $J=0.9 \mathrm{~Hz}$, $3 \mathrm{H}) ;{ }^{13} \mathrm{C}$ NMR (75 MHz, $\mathrm{CDCl}_{3}$ ): $\delta$ 140.3, 137.9, 130.4, 130.0, 129.1, 128.6, 128.1, 126.7, 67.0, 62.9, 51.0, 32.6, 26.4, 25.7, 17.8; IR (neat, $\mathrm{cm}^{-1}$ ): 2927, 2795, 1494, 1454, 1381, 1125, 1058, 1025, 967, 870, 809, 736, 698; Analytical HPLC (column: CHIRALCEL OD-H (0.46 cm $\times 25 \mathrm{~cm}$ ), eluent: hexane $/ i-\mathrm{PrOH}=1000 / 1$, flow rate: $0.3 \mathrm{~mL} / \mathrm{min}$, detection: UV $254 \mathrm{~nm}$, temperature: $\mathrm{rt}$ ) $t_{R}=22.0 \mathrm{~min}$ for $(S)$-isomer, $23.3 \mathrm{~min}$ for $(R)$-isomer; Optical rotation value: $[\alpha]_{\mathrm{D}}{ }^{27}=-36.4(c=1.51, \mathrm{EtOH})$ for $(R)$-isomer $(>98 \% e e),[\alpha]_{\mathrm{D}}{ }^{29}=$ $+35.9\left(c=2.03, \mathrm{CHCl}_{3}\right)$ for $(S)$-isomer $(>98 \% e e)$. 
(3E,7Z)-1,1-Dibenzyl-3,7-dimethyl-2,5,6,9-tetrahydro-1 $H$-azoninium bromide (1d)

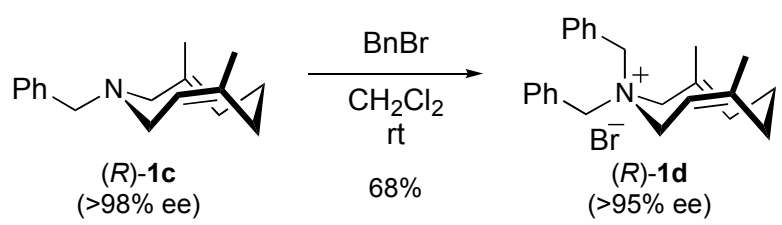

To a solution of $(R)-\mathbf{1 c}\left(119 \mathrm{mg}, 0.493 \mathrm{mmol},>98 \%\right.$ ee) in dry $\mathrm{CH}_{2} \mathrm{Cl}_{2}(0.5 \mathrm{~mL})$ at room temperature was added benzyl bromide $(0.53 \mathrm{~mL}, 4.88 \mathrm{mmol})$. The reaction mixture was stirred for $24 \mathrm{~h}$ at that temperature, concentrated in vacuo and the residue was purified by silica gel chromatography $\left(\mathrm{CHCl}_{3} / \mathrm{MeOH}=20 / 1\right.$ to $\left.10 / 1\right)$ to afford $137 \mathrm{mg}$ of ammonium salt of $(R)-\mathbf{1 d}(68 \%,>95 \%$ ee determined by chiral HPLC analysis). ${ }^{1} \mathrm{H}$ NMR (300 MHz, $\left.\mathrm{CDCl}_{3}\right): \delta$ 7.92-7.88 (m, 2H), 7.60-7.57 (m, 2H), 7.52-4.49 (m, 3H), 7.44-7.39 (m, 3H), $5.92(\mathrm{~d}, J=12.6 \mathrm{~Hz}, 1 \mathrm{H}), 5.86(\mathrm{~d}, J=12.6 \mathrm{~Hz}, 1 \mathrm{H}), 5.83(\mathrm{dd}, J=12.3,3.3 \mathrm{~Hz}$, $1 \mathrm{H}), 5.12(\mathrm{dd}, J=12.3,3,3 \mathrm{~Hz}, 1 \mathrm{H}), 4.95(\mathrm{dd}, J=11.4,3.9 \mathrm{~Hz}, 1 \mathrm{H}), 4.74(\mathrm{~d}, J=12.6 \mathrm{~Hz}, 1 \mathrm{H}), 4.19$ (d, $J=$ $12.6 \mathrm{~Hz}, 1 \mathrm{H}), 3.84$ (d, $J=12.6 \mathrm{~Hz}, 1 \mathrm{H}), 3.79$ (d, $J=12.6 \mathrm{~Hz}, 1 \mathrm{H}), 3.23(\mathrm{dd}, J=12.3,12.3 \mathrm{~Hz}, 1 \mathrm{H})$, 2.17-2.10 (m, 1H), 2.05-1.90 (m, 2H), $1.86(\mathrm{~s}, 3 \mathrm{H}), 1.82(\mathrm{~s}, 3 \mathrm{H}), 1.70-1.55(\mathrm{~m}, 1 \mathrm{H}) ;{ }^{13} \mathrm{C}$ NMR $(75 \mathrm{MHz}$, $\left.\mathrm{CDCl}_{3}\right): 142.6,141.4,134.0,133.9,130.6,130.3,129.3,129.0,128.2,128.0,117.4(2 \mathrm{C}), 66.2,64.1,64.0$, 58.9, 32.3, 26.7, 26.5, 19.6; Analytical HPLC (column: CHIRALCEL OD-RH (0.46 cm $\times 15 \mathrm{~cm}$ ), eluent: $\mathrm{MeOH} / 0.1 \mathrm{M}$ aq $\mathrm{KPF}_{6}=90 / 10$, flow rate: $0.5 \mathrm{~mL} / \mathrm{min}$, detection: $\mathrm{UV} 230 \mathrm{~nm}$, temperature: $\left.\mathrm{rt}\right) t_{R}=7.4 \mathrm{~min}$ for $(S)$-isomer, $8.6 \mathrm{~min}$ for $(R)$-isomer.

(Z)-3,7-dimethyl-1-( $p$-toluenesulfonyl)-2,3,4,5,6,9-hexahydro-1 $H$-azonin-4-ol (7)

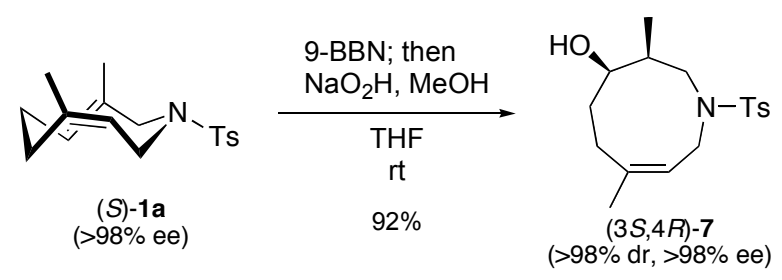

To a solution of 9-BBN $(0.31 \mathrm{~mL}$ of $0.5 \mathrm{M}$ in THF, $0.156 \mathrm{mmol})$ in dry THF $(2 \mathrm{~mL})$ at room temperature was added $(S)$-1a (19.8 mg, $0.0648 \mathrm{mmol},>98 \%$ ee) dissolved in dry THF $(3 \mathrm{~mL})$. After the solution was stirred for $2 \mathrm{~h}$ at that temperature, the organoborane was immediately oxidized by adding $\mathrm{MeOH}(0.5 \mathrm{~mL})$, $6 \mathrm{~N} \mathrm{NaOH}$ (aq) $(0.5 \mathrm{~mL})$ and $30 \% \mathrm{H}_{2} \mathrm{O}_{2}(5.0 \mathrm{~mL})$ at $0{ }^{\circ} \mathrm{C}$. The mixture was stirred at $40{ }^{\circ} \mathrm{C}$ for $10 \mathrm{~min}$ to ensure complete oxidation, then cooled to room temperature. The reaction was quenched with aq satd $\mathrm{NaHCO}_{3}$ and extracted with $\mathrm{Et}_{2} \mathrm{O}$. The combined organic phase was dried over $\mathrm{Na}_{2} \mathrm{SO}_{4}$, filtered and the solvent was removed in vacuo. The crude product was purified by silica gel chromatography (hexane/AcOEt $=1 / 1)$ to afford $19.4 \mathrm{mg}$ of $(3 S, 4 R)-7\left(92 \%,>98 \% \mathrm{dr}\right.$ analyzed by ${ }^{1} \mathrm{H} \mathrm{NMR},>98 \%$ ee determined by chiral HPLC analysis) as a white solid. ${ }^{1} \mathrm{H}$ NMR $\left(300 \mathrm{MHz}, \mathrm{CDCl}_{3}\right): \delta 7.71(\mathrm{~d}, J=8.4 \mathrm{~Hz}$, 2H), 7.31 (d, $J=8.4 \mathrm{~Hz}, 2 \mathrm{H}), 5.50$ (t, $J=7.8 \mathrm{~Hz}, 1 \mathrm{H}), 4.02(\mathrm{dt}, J=9.9,2.7 \mathrm{~Hz}, 1 \mathrm{H}), 3.92$ (dd, $J=15.6,7.8$ $\mathrm{Hz}, 1 \mathrm{H}), 3.44$ (dd, $J=15.3,6.0 \mathrm{~Hz}, 1 \mathrm{H}), 3.32$ (dd, $J=15.6,7.8 \mathrm{~Hz}, 1 \mathrm{H}), 2.81$ (dd, $J=15.3,11.6 \mathrm{~Hz}, 1 \mathrm{H}$ ), $2.43(\mathrm{~s}, 3 \mathrm{H}), 2.35(\mathrm{~m}, 2 \mathrm{H}), 2.06(\mathrm{~m}, 1 \mathrm{H}), 1.91(\mathrm{~m}, 1 \mathrm{H}), 1.78(\mathrm{~s}, 3 \mathrm{H}), 1.71(\mathrm{~m}, 1 \mathrm{H}), 1.55(\mathrm{br}, 1 \mathrm{H}), 0.92(\mathrm{~d}, J$ $=6.9 \mathrm{~Hz}, 3 \mathrm{H}) ;{ }^{13} \mathrm{C} \mathrm{NMR}\left(75 \mathrm{MHz}, \mathrm{CDCl}_{3}\right): \delta 143.3,143.0,136.3,129.8,127.2,121.2,70.2,55.3,49.2$, 
40.2, 29.0, 28.8, 23.1, 21.6, 12.1; IR (neat, $\mathrm{cm}^{-1}$ ): 3503, 2931, 2872, 1473, 1449, 1334, 1156, 1090, 993, 755, 653; Analytical HPLC (column: CHIRALCEL OD-H (0.46 cm x $25 \mathrm{~cm}$ ), eluent: hexane/iPrOH = 1/1, flow rate: $0.3 \mathrm{~mL} / \mathrm{min}$, detection: UV $254 \mathrm{~nm}$, temperature: $\mathrm{rt}) t_{R}=13.7 \mathrm{~min}$ for $(3 R, 4 S)$-isomer, $15.9 \mathrm{~min}$ for $(3 S, 4 R)$-isomer; Anal. Calcd. for $\mathrm{C}_{17} \mathrm{H}_{25} \mathrm{NO}_{3} \mathrm{~S}$ : C, 63.13; H, 7.79; N, 4.33; S, 9.91. Found: C, 63.66; H, $7.72 ; \mathrm{N}, 3.80 ; \mathrm{S}, 8.38$.

4-isopropenyl-3-methyl-1-(p-toluenesulfonyl)-3-vinyl-pyrrolidine (8)

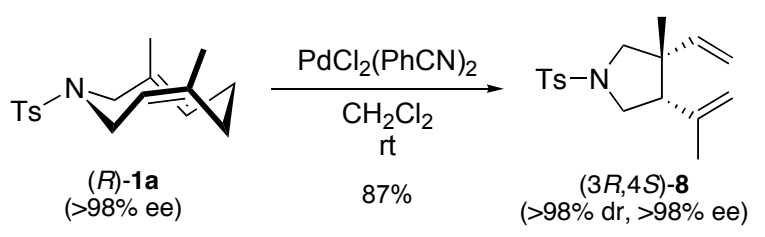

To a solution of $(R)-\mathbf{1 a}\left(19.9 \mathrm{mg}, 0.0652 \mathrm{mmol},>98 \%\right.$ ee) in dry $\mathrm{CH}_{2} \mathrm{Cl}_{2}(3 \mathrm{~mL})$ at room temperature was added $\mathrm{PdCl}_{2}\left(\mathrm{PhCN}_{2}(2.5 \mathrm{mg}, 0.00652 \mathrm{mmol})\right.$. The resulting solution was stirred for $2 \mathrm{~h}$ at that temperature, then filtrated through a pad of Celite. The solvent was removed in vacuo and the crude product was purified by silica gel chromatography (hexane/AcOEt $=10 / 1$ to $3 / 1$ ) to afford $17.3 \mathrm{mg}$ of $(3 R, 4 S)-8\left(87 \%,>98 \% \mathrm{dr}\right.$ analyzed by ${ }^{1} \mathrm{H}$ NMR, $>98 \%$ ee determined by chiral HPLC analysis). The stereochemistry of $\mathbf{8}$ was determined by NOE experiment as shown in Figure I. ${ }^{1} \mathrm{H}$ NMR $(300 \mathrm{MHz}$, $\left.\mathrm{CDCl}_{3}\right): \delta 7.74(\mathrm{~d}, J=8.1 \mathrm{~Hz}, 2 \mathrm{H}), 7.33(\mathrm{~d}, J=8.1 \mathrm{~Hz}, 2 \mathrm{H}), 5.62(\mathrm{dd}, J=17.4,10.8 \mathrm{~Hz}, 1 \mathrm{H}), 4.96(\mathrm{dd}, J=$ $10.8,0.9 \mathrm{~Hz}, 1 \mathrm{H}), 4.91$ (dd, $J=17.4,0.9 \mathrm{~Hz}, 1 \mathrm{H}), 4.83-4.81$ (dq, $J=1.5,1.5 \mathrm{~Hz}, 1 \mathrm{H}), 4.60$ (d, $J=1.5 \mathrm{~Hz}$, 1H), 3.51 (dd, $J=10.1,7.7 \mathrm{~Hz}, 1 \mathrm{H}), 3.41$ (d, $J=9.9 \mathrm{~Hz}, 1 \mathrm{H}), 3.34$ (dd, $J=10.1,8.5 \mathrm{~Hz}, 1 \mathrm{H}), 3.06$ (d, $J=$ $9.9 \mathrm{~Hz}, 1 \mathrm{H}), 2.44(\mathrm{~s}, 3 \mathrm{H}), 2.36(\mathrm{dd}, J=8.5,7.7 \mathrm{~Hz}, 1 \mathrm{H}), 1.60(\mathrm{~d}, J=1.5 \mathrm{~Hz}, 3 \mathrm{H}), 1.06(\mathrm{~s}, 3 \mathrm{H}) ;{ }^{13} \mathrm{C} \mathrm{NMR}$ $\left(75 \mathrm{MHz}, \mathrm{CDCl}_{3}\right): \delta 143.3,141.7,139.9,134.2,129.6,127.4,113.9,113.6,58.1,55.1,50.6,47.0,23.8$, 23.0, 21.7; IR (neat, $\mathrm{cm}^{-1}$ ): 2965, 2924, 1640, 1598, 1453, 1346, 1156, 1094, 1055, 663; Analytical HPLC (column: CHIRALCEL OD-H $(0.46 \mathrm{~cm} \times 25 \mathrm{~cm})$, eluent: hexane $/ \mathrm{PrOH}=30 / 1$, flow rate: $0.5 \mathrm{~mL} / \mathrm{min}$, detection: UV $254 \mathrm{~nm}$, temperature: $\mathrm{rt}) t_{R}=19.1 \mathrm{~min}$ for $(-)-(3 R, 4 S)$-isomer, $20.5 \mathrm{~min}$ for $(+)-(3 S, 4 R)$-isomer; Optical rotation value: $[\alpha]_{\mathrm{D}}{ }^{26}=-5.3\left(c=1.35, \mathrm{CHCl}_{3}\right)$ for $(3 R, 4 S)$-isomer $(>98 \%$ ee $)$; Anal. Calcd. for $\mathrm{C}_{17} \mathrm{H}_{23} \mathrm{NO}_{2} \mathrm{~S}$ : C, 66.85; H, 7.59; N, 4.59; S, 10.50. Found: C, 66.58; H, 7.44; N, 4.41; S, 10.32 .

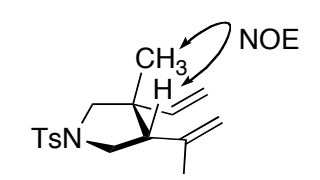

Figure I. NOE analysis of 8 


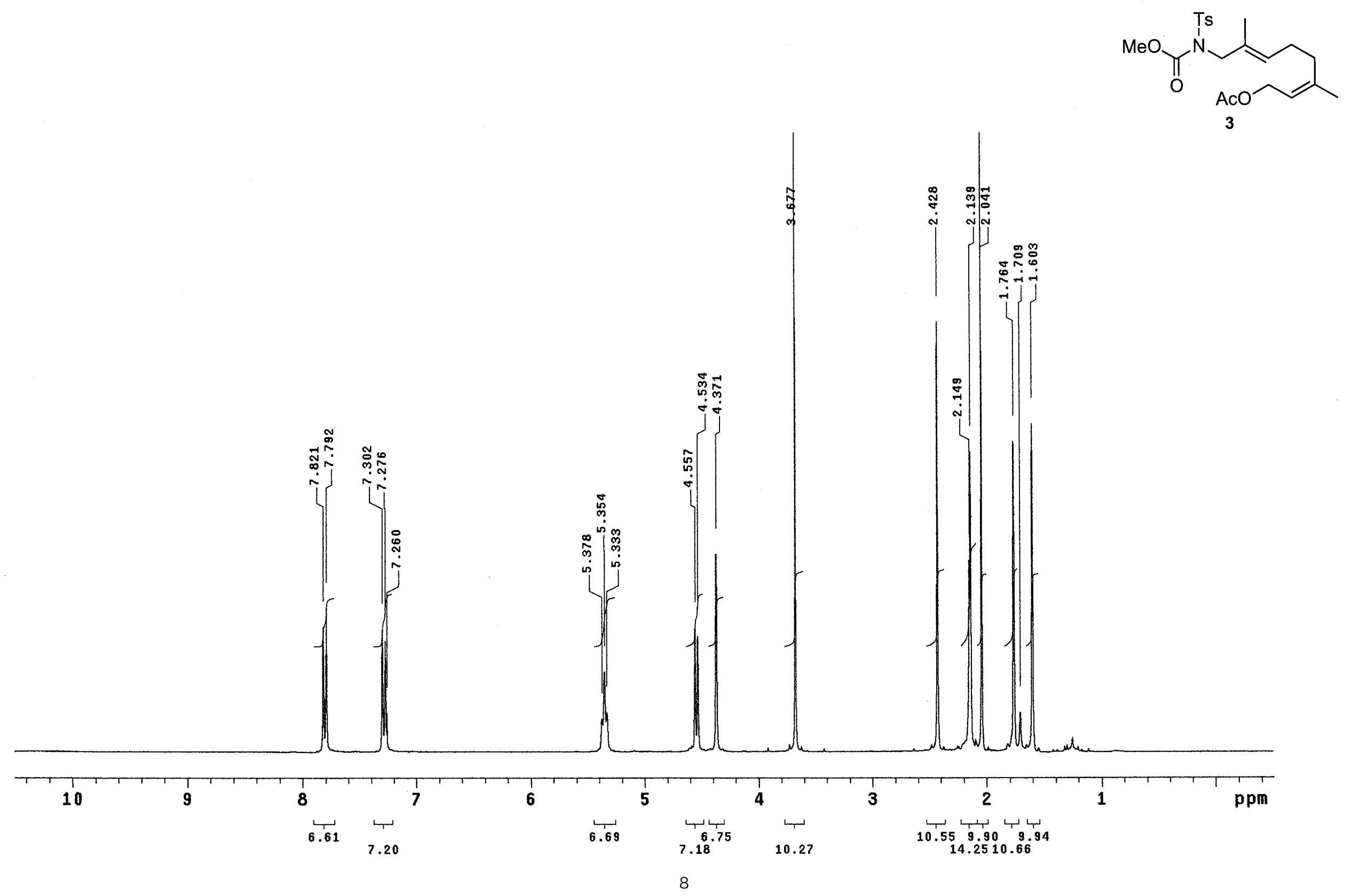



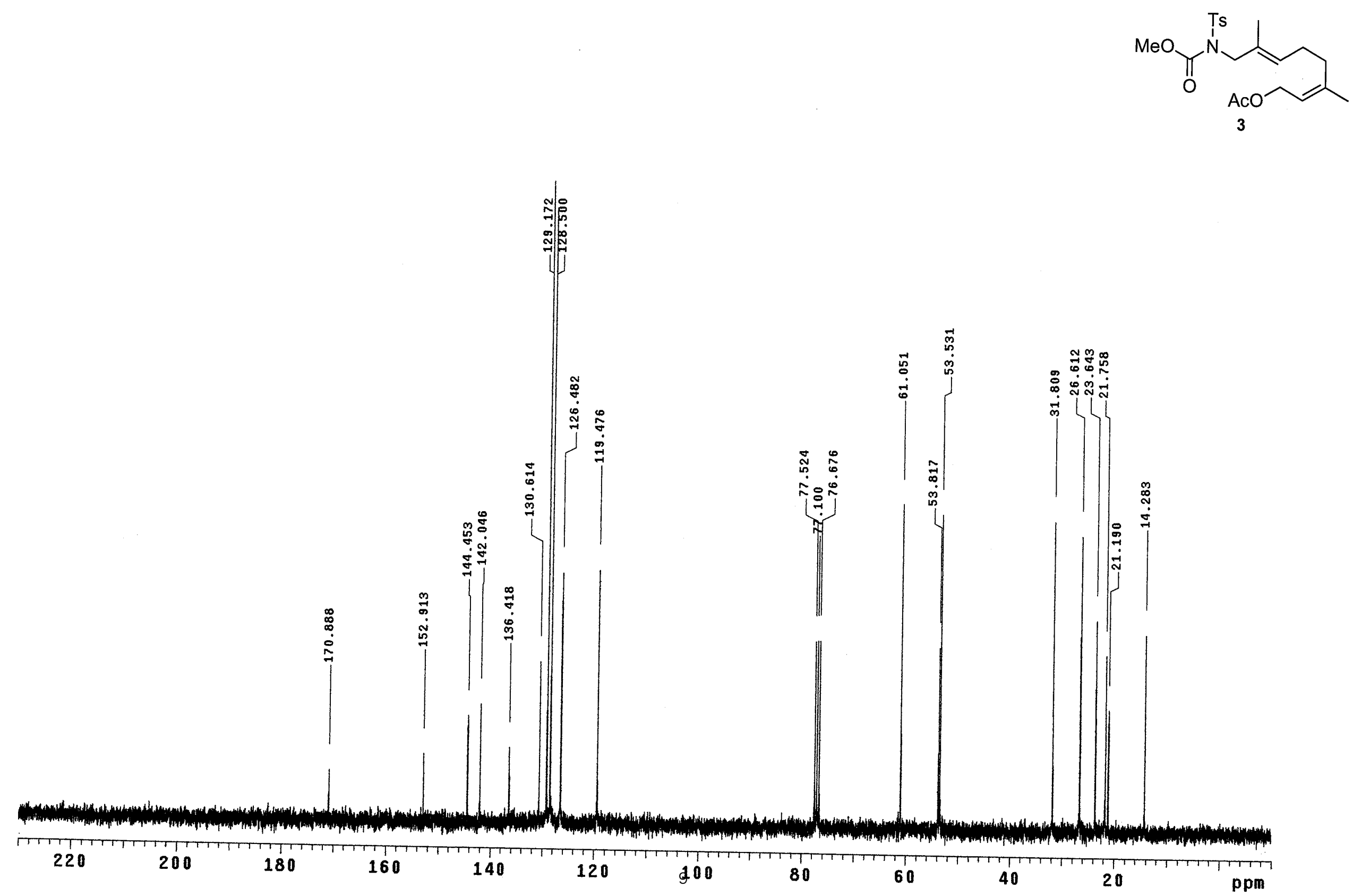


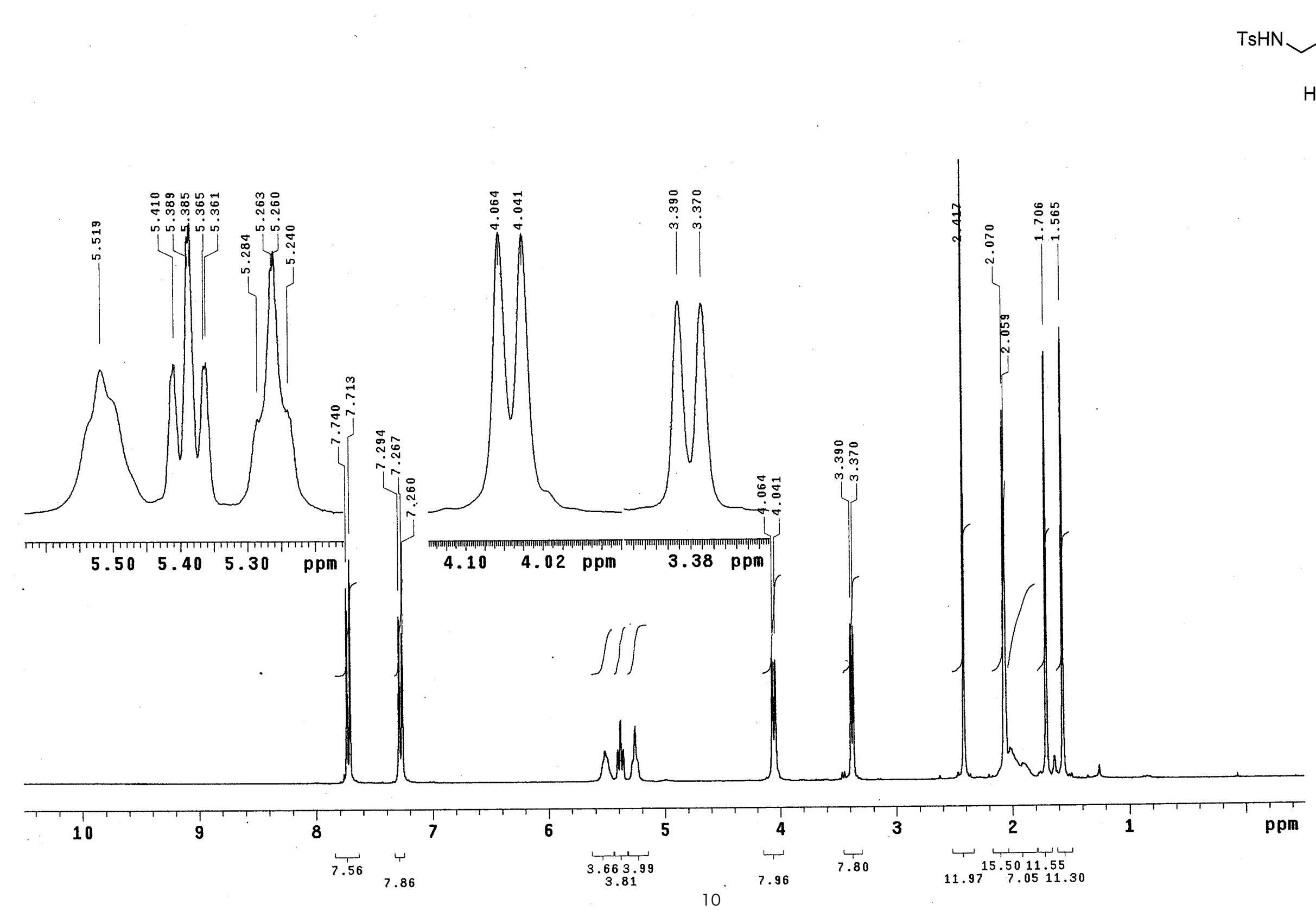




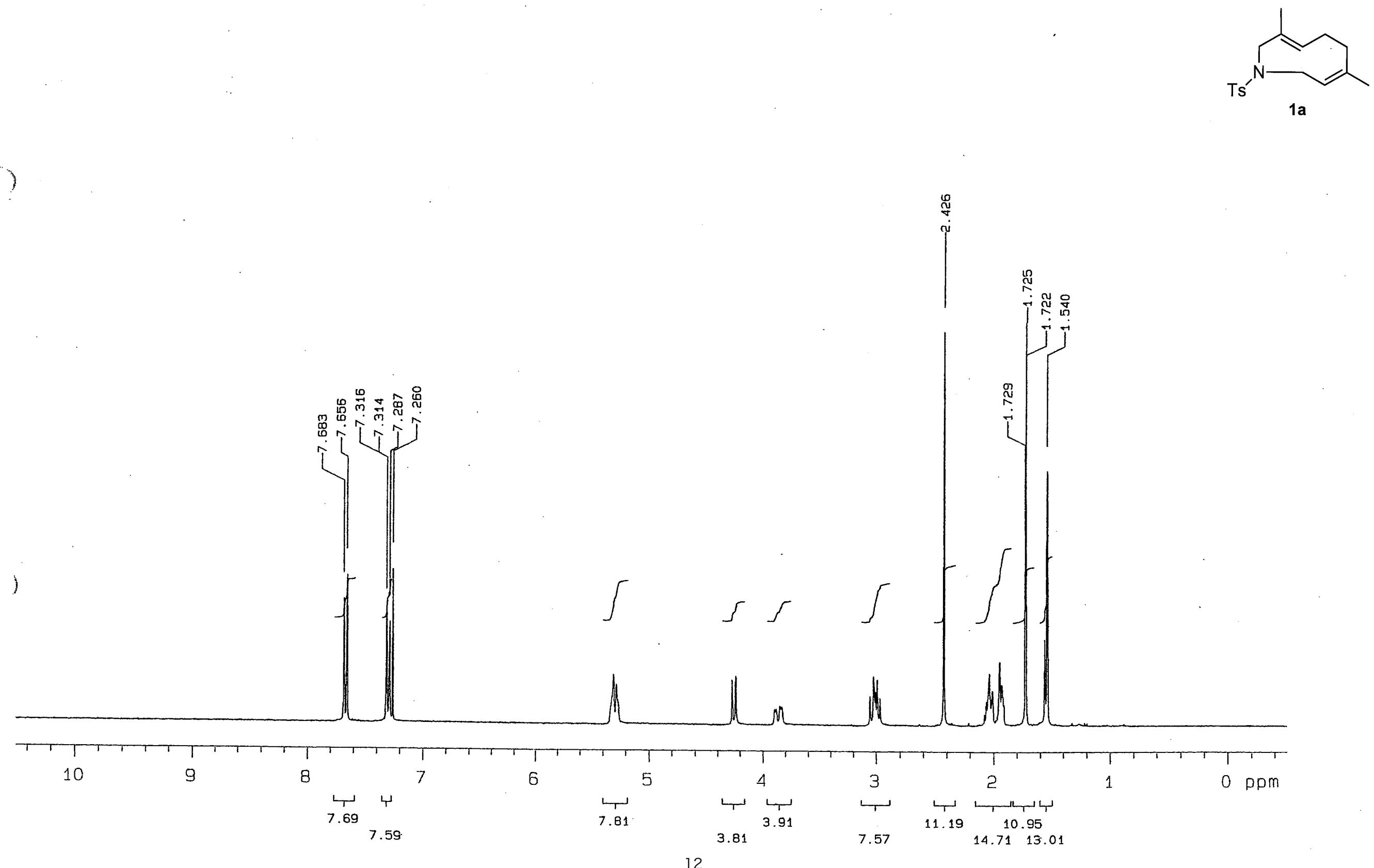


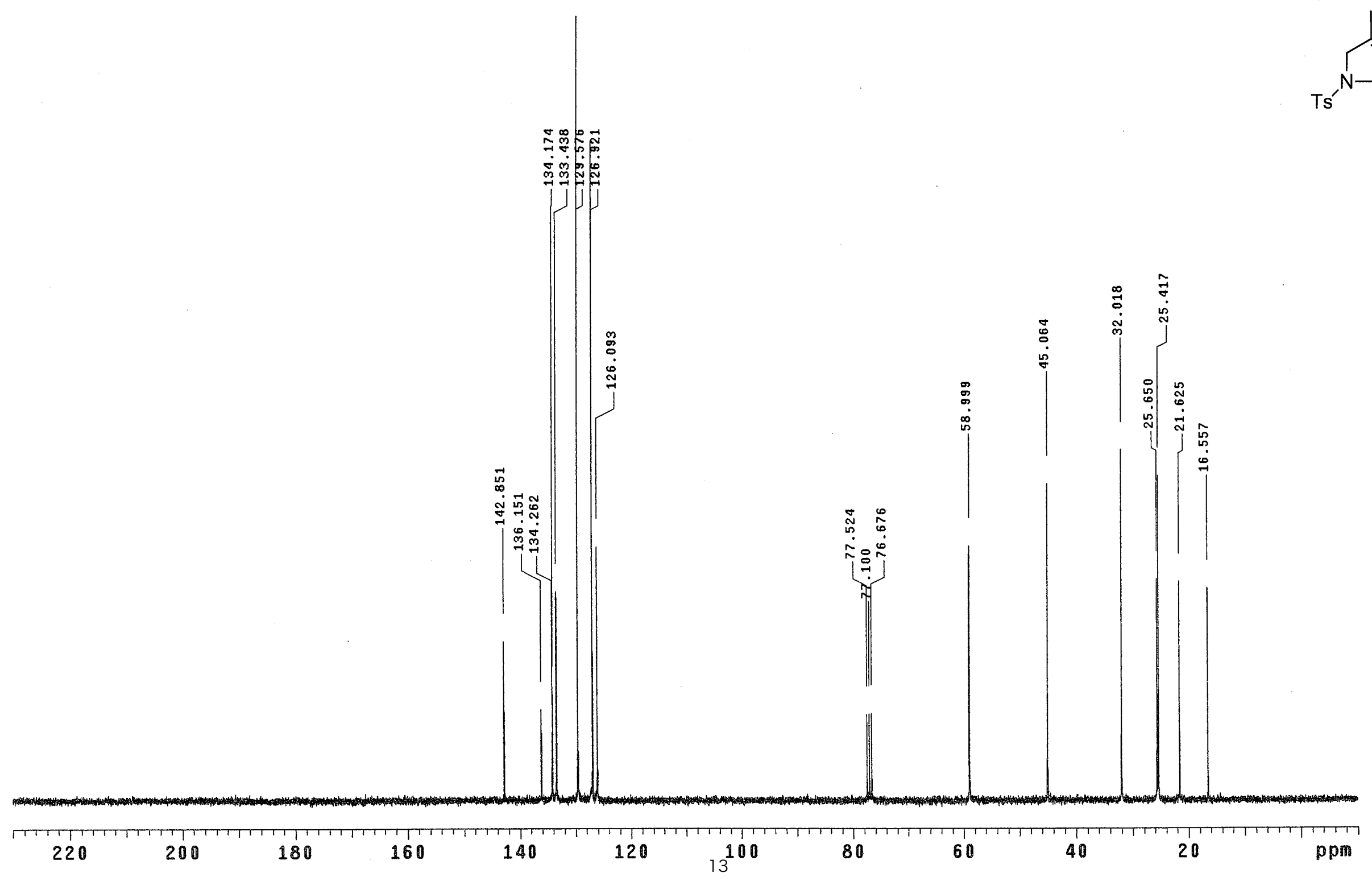




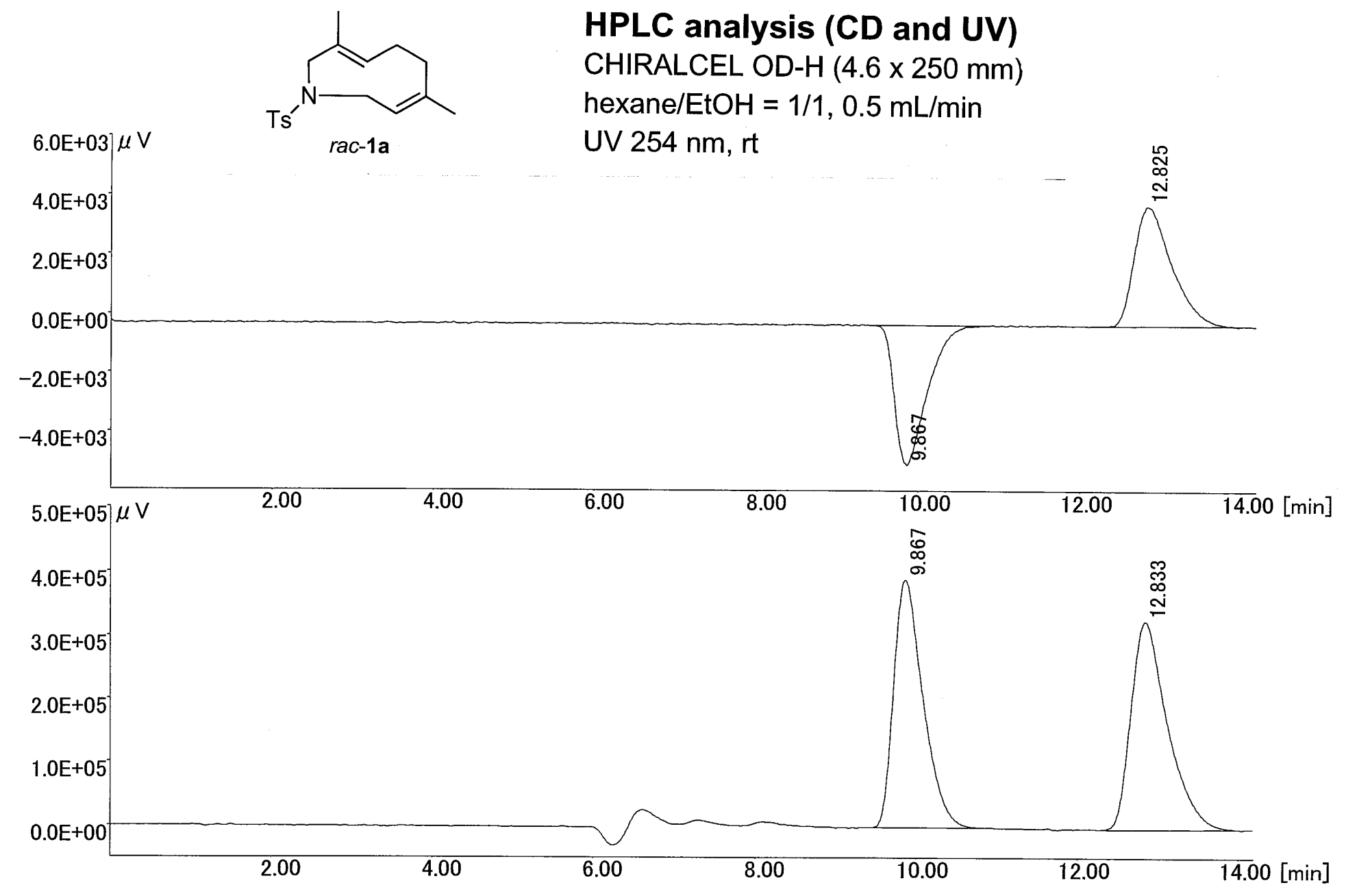

ファイル名：RN-259-prd-0DH153. CH1

コメント:

Vial \#= 1 Rack \#= 1

注入日：6-Aug-2004 18:20:26

現在日時：9-Aug-2005 15:32:34

ユーザー: NISHIKAWA

グループ： TOMOOKA

システムプログラム:RN-TYUUINNKANN

\# ピーク名 Rt 面積 $[\mu \mathrm{V}$. Sec] 定量値 面積\%

$\begin{array}{lllll}1 & 9.867 & 124514.500 & 0.000 & 48.90 \\ 2 & 12.825 & 130100.856 & 0.000 & 51.10\end{array}$

ピーク総面積 $=254615.356$ [ $\mu \mathrm{V} . \mathrm{Sec}]$

ファイル名：RN-259-prd-0DH153. CH2

コメント:

Vial \# $=1$ Rack \# $=1$

注入日：6-Aug-2004 18:20:26

現在日時：9-Aug-2005 15:32:34

ユーザー：NISHIKAWA

グループ：TOMOOKA

ジステムプログラム:RN-TYUUI INKANN

\# ピーク名 Rt 面積 $[\mu \mathrm{V} . \mathrm{Sec}]$ 定量値 面積\%

$\begin{array}{llrll}1 & 9.867 & 9845730.109 & 0.000 & 48.86 \\ 2 & 12.833 & 10306229.250 & 0.000 & 51.14\end{array}$

ピーク総面積 $=20151959.359$ [ $\mu \mathrm{V} . \mathrm{Sec}]$ 


\section{CD and UV spectra}

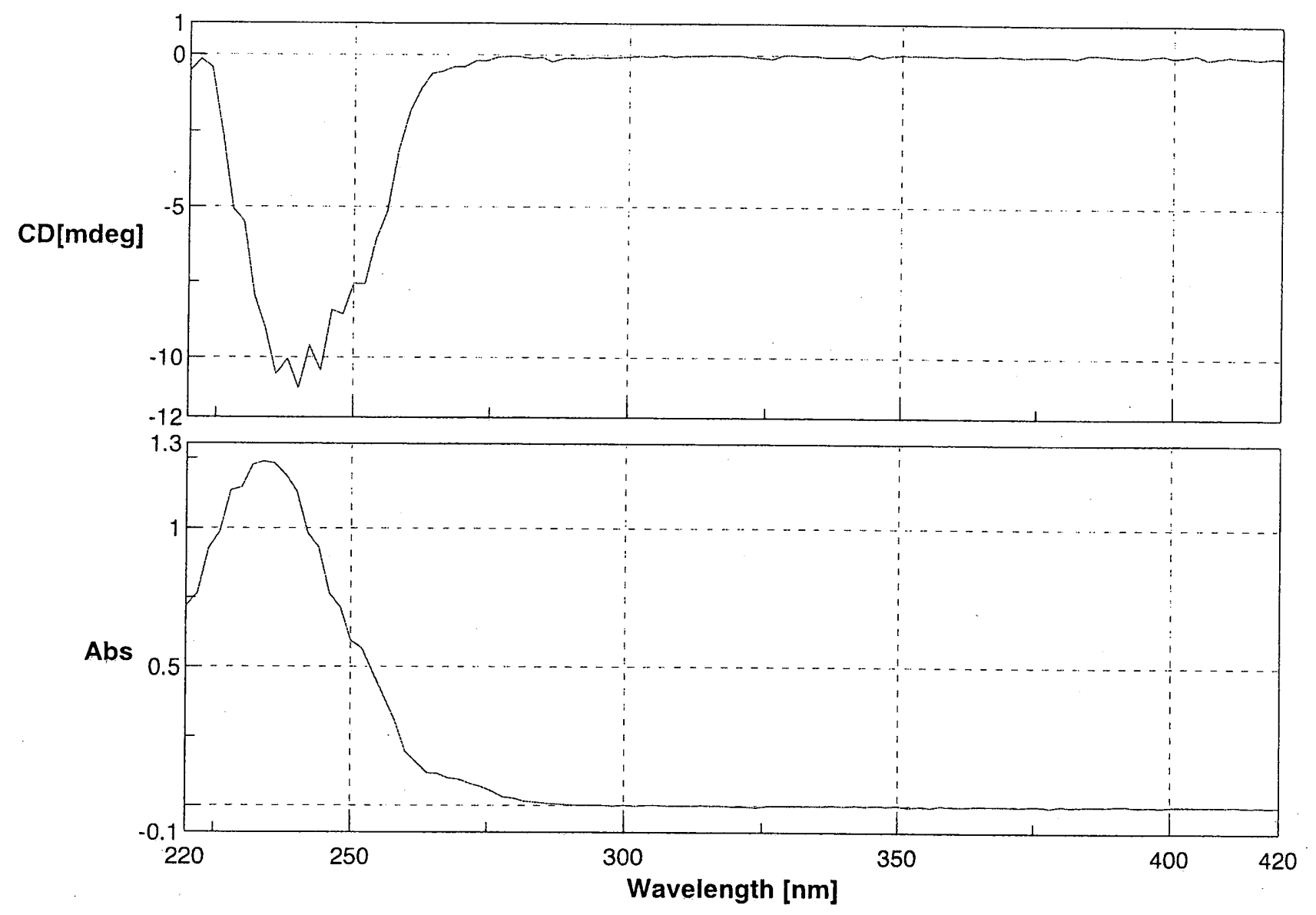

旦時

機種名

シリアル番号

試料名

測定者

コメシト
2004/12/09 15:20

dimethyl-CD-1. jws

003460875

CD/UV Spectra $t_{\mathrm{R}}=9.9 \mathrm{~min}$ for $(R)$-isomer

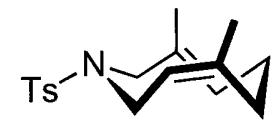

$(R)-1 \mathrm{a}$ 

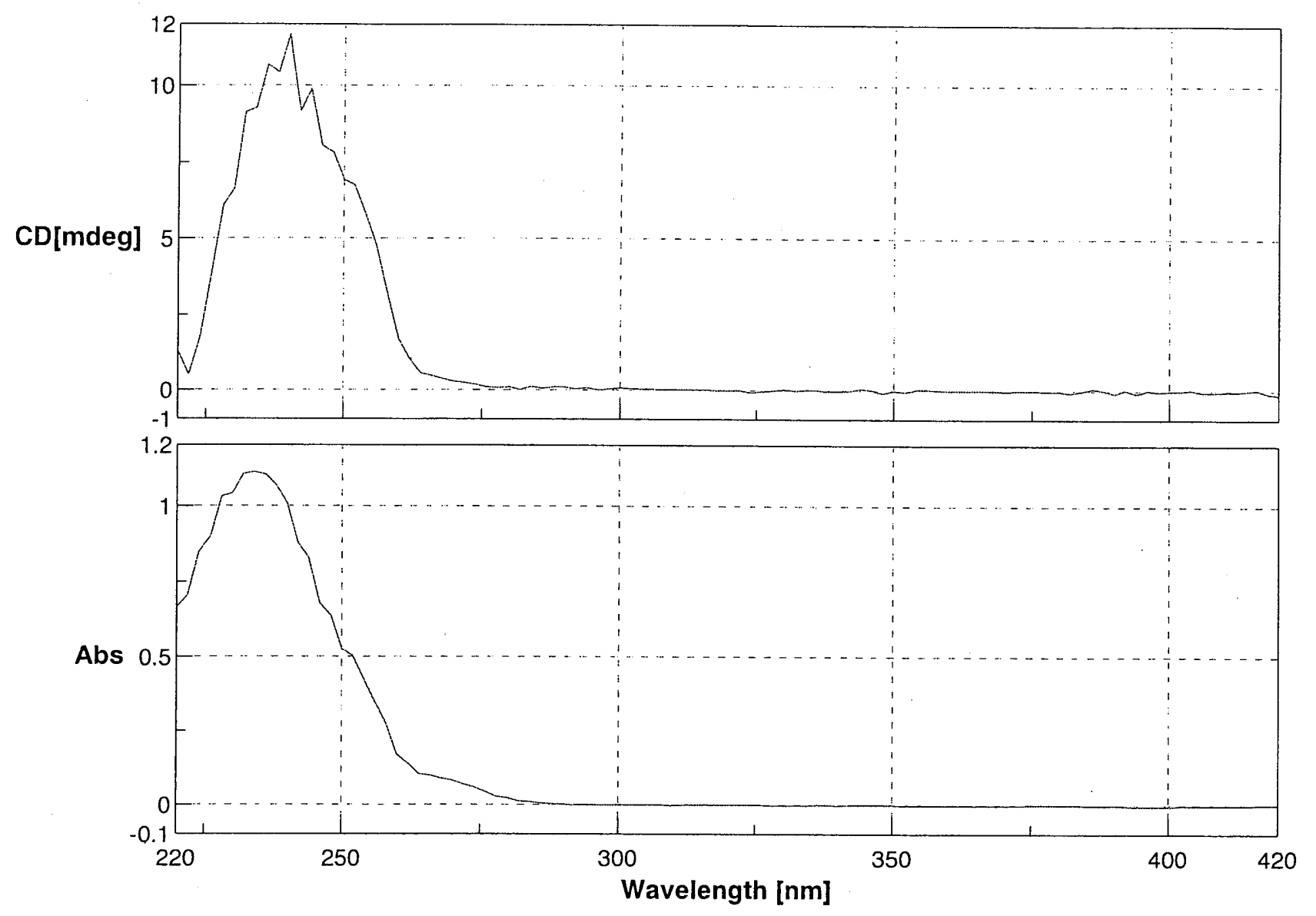

旦時

機種名

シリアル番号

試料名

測定者

コメント
2004/12/09 15:24

dimethyl-CD-2. jws

003460875

CD/UV Spectra $t_{R}=12.8 \mathrm{~min}$ for $(S)$-isomer

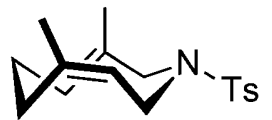

(S)-1a 

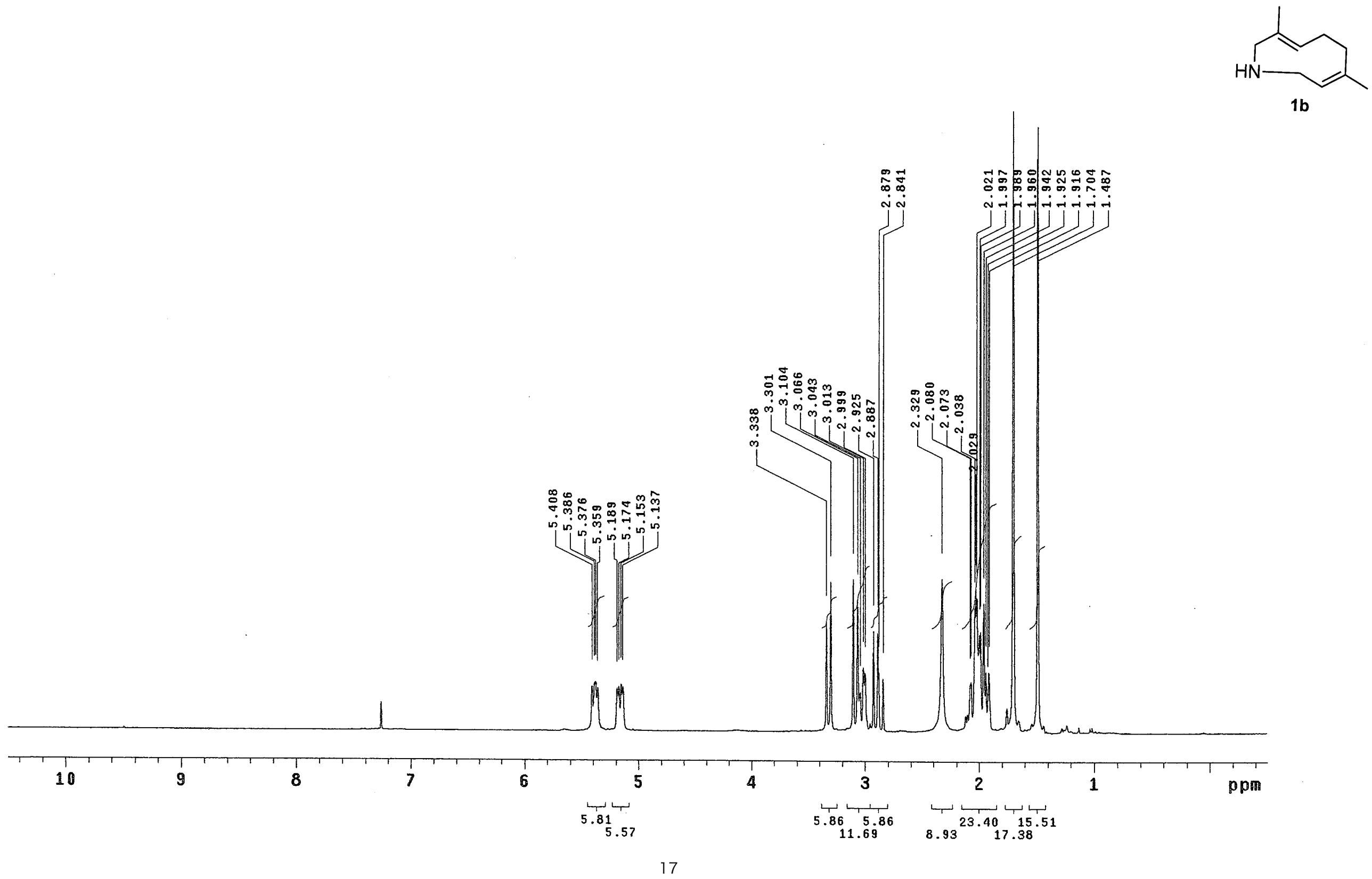


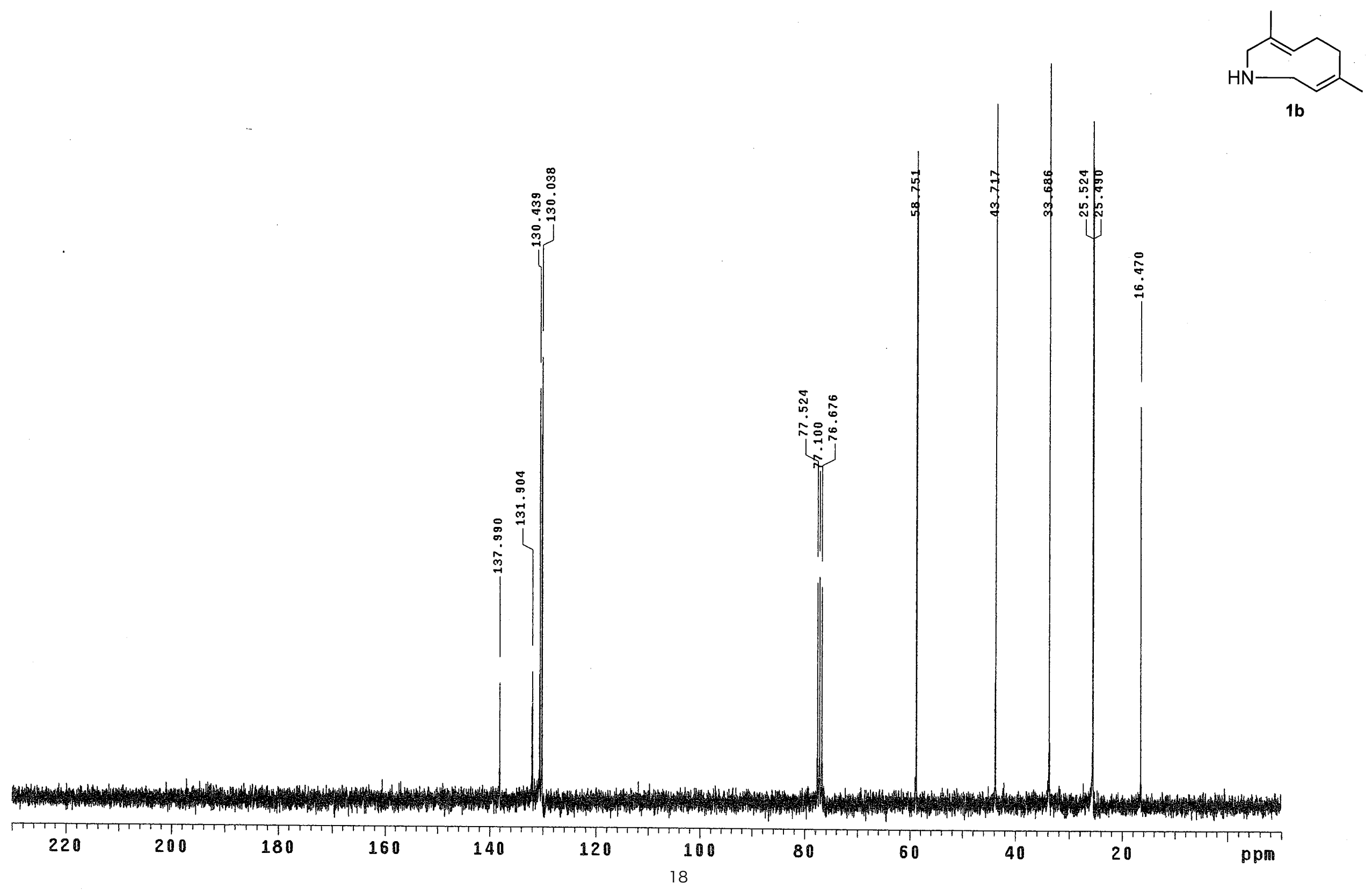




$$
P 11]
$$




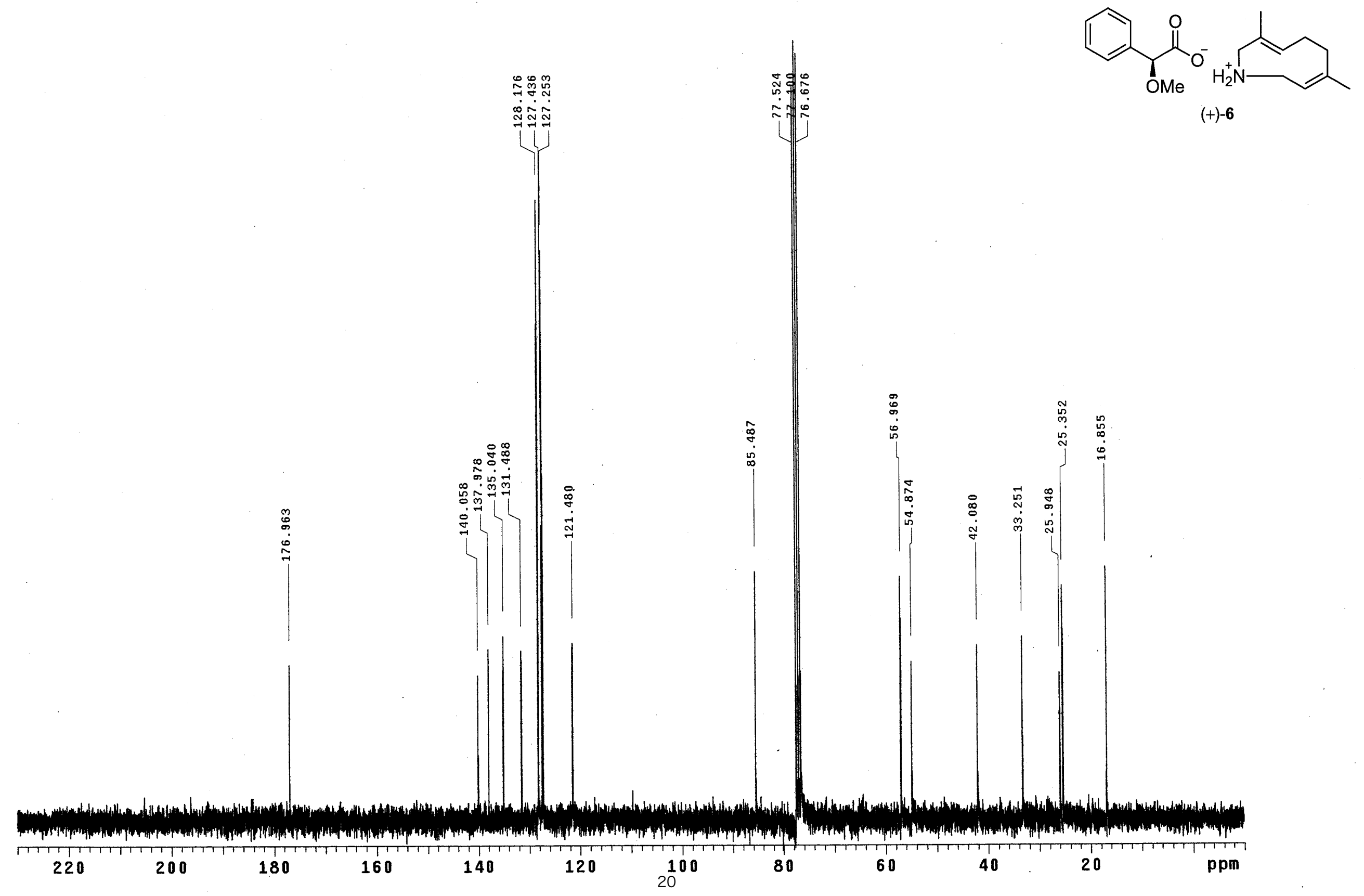




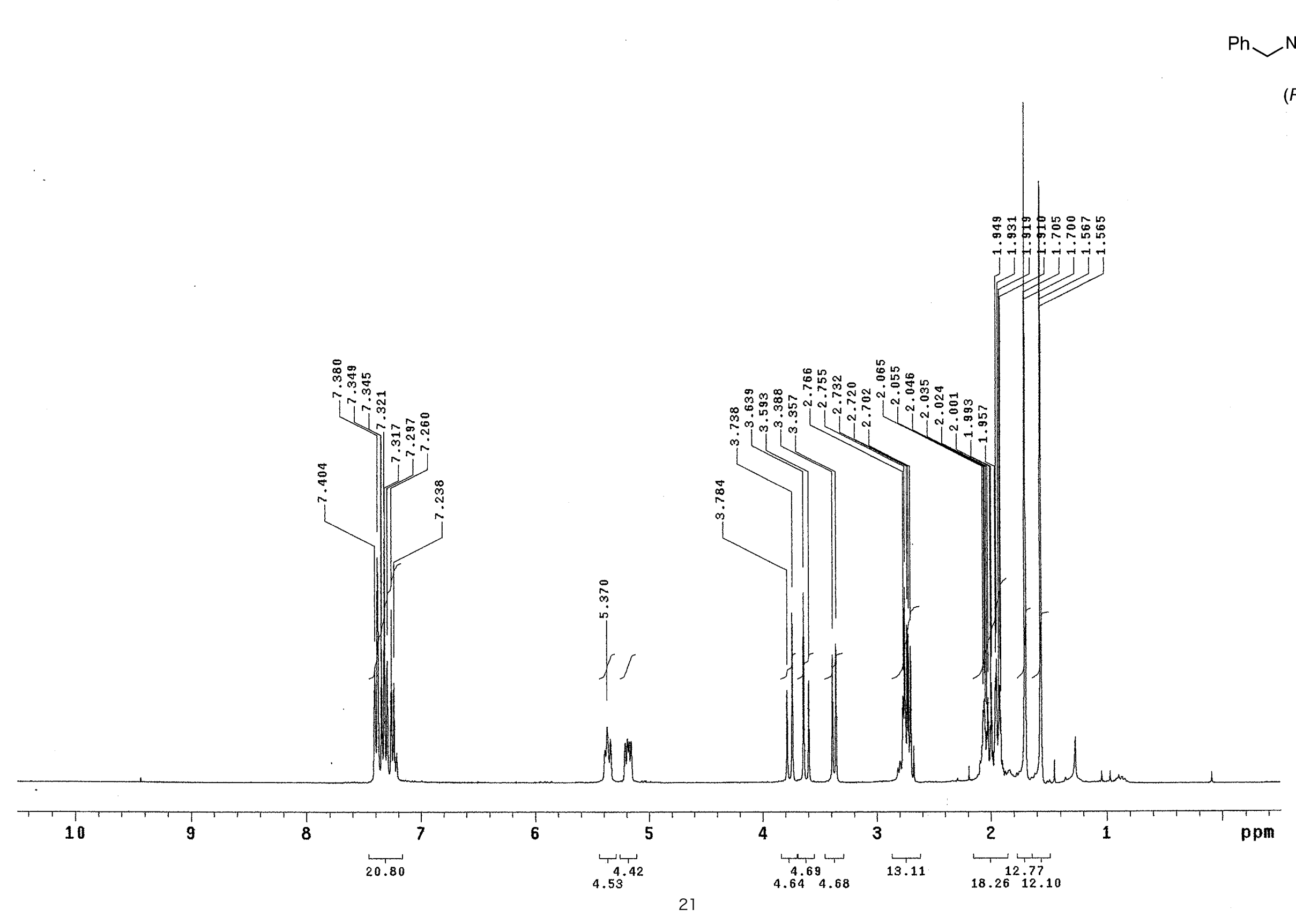


$\mathrm{N}^{\mathrm{N}}$

(R)-1c

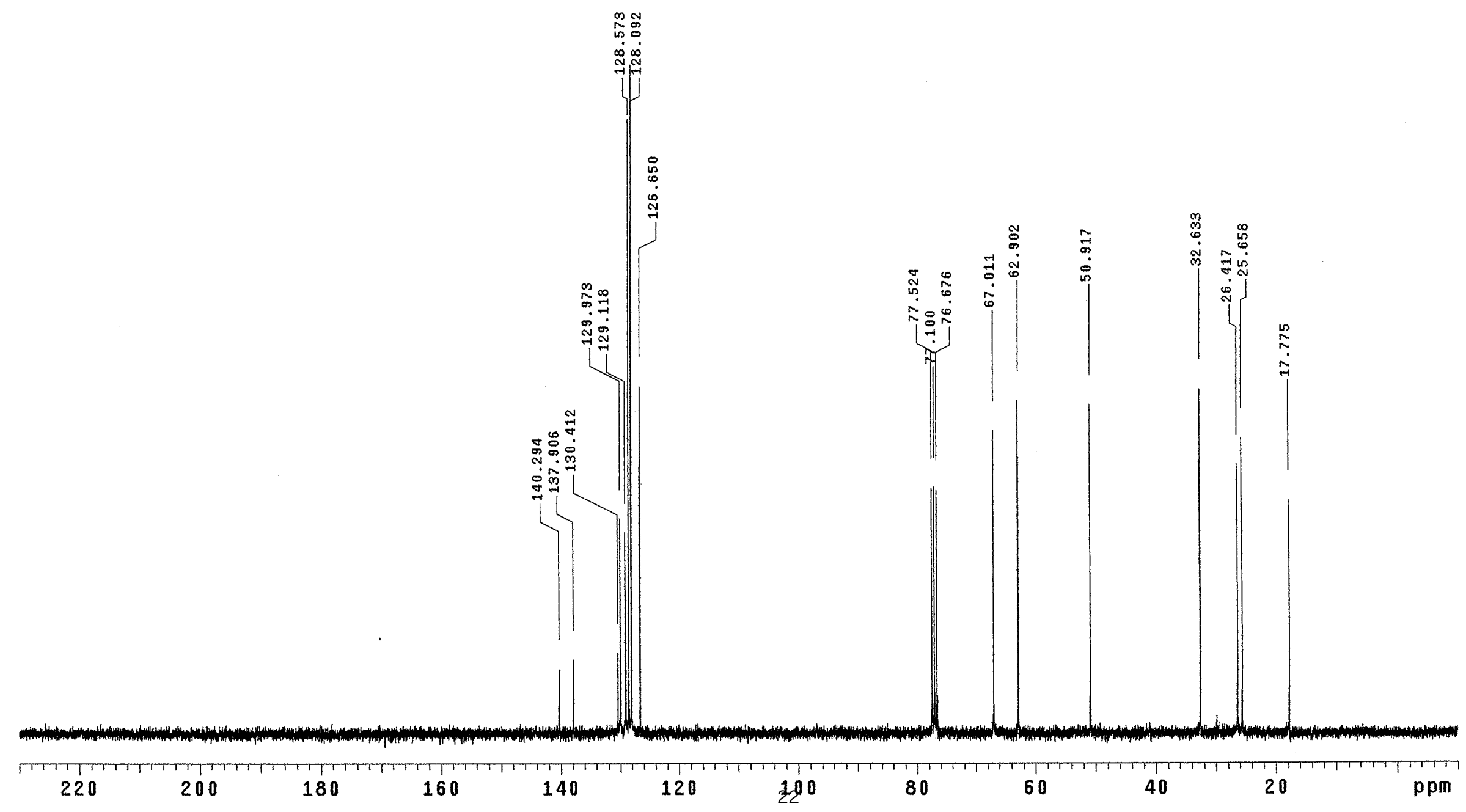




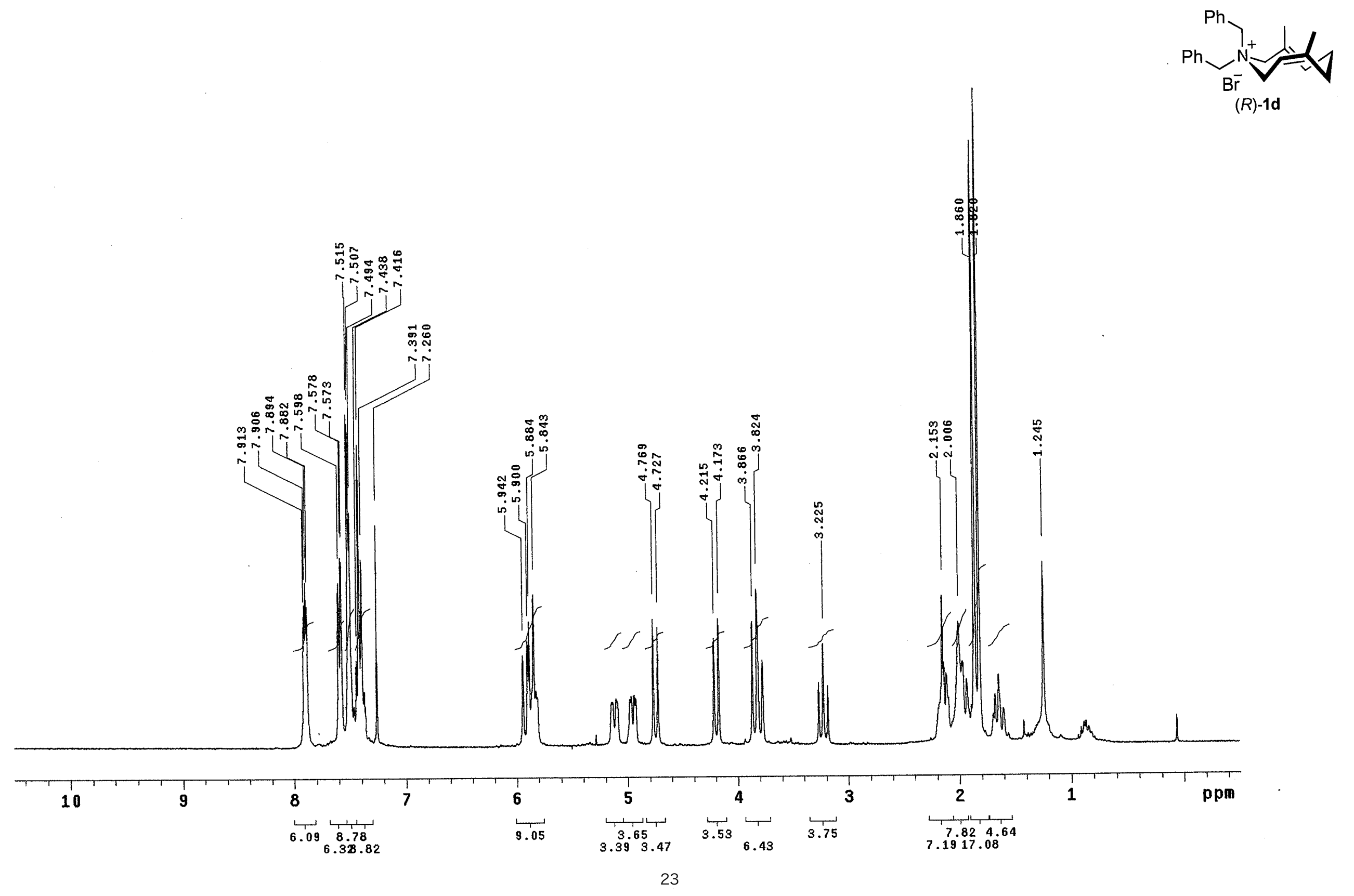



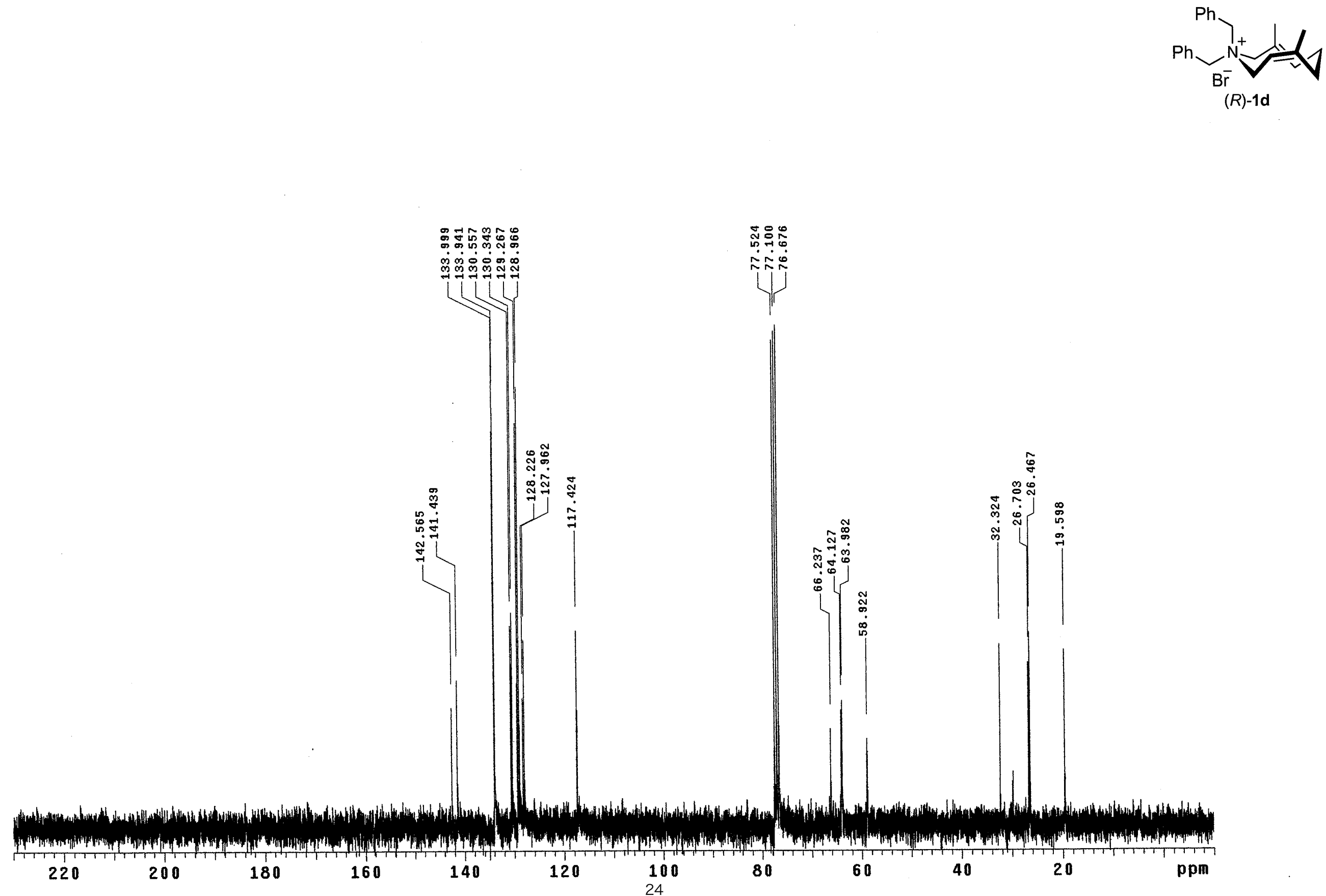


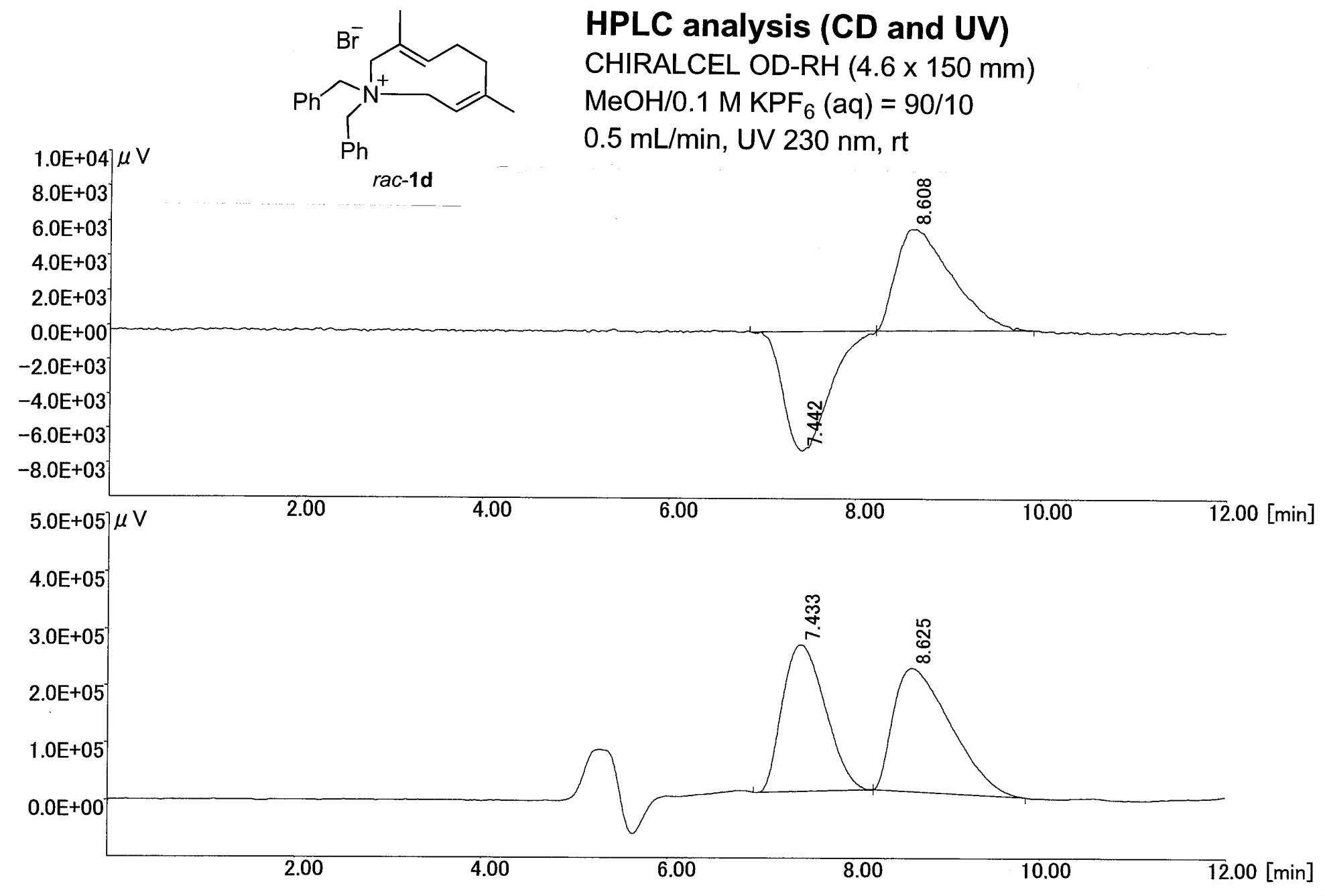

ファイル名: KU518-2-001. CH1

コメント:

Vial \# $=1$ Rack \#= 1

注入星: 12-Jun-2004 12:39:02

現在昌時: 9-Aug-2005 20:10:42

ユーザー：UEHARA

グループ： TOMOOKA

システムプログラム:MEOH90-KPF10-2-UV230

\# ピーク名 Rt 面積 $[\mu \mathrm{V} . \mathrm{Sec}] \quad$ 面積\%

$\begin{array}{llll}1 & 7.442 & 224746.333 & 47.59\end{array}$

ピーク総面積 $=472212.533$ [ $\mu \mathrm{V} . \mathrm{Sec}]$

ファイル名: KU518-2-001. CH2

コメント:

Vial \# = 1 Rack \#= 1

注入旦：12-Jun-2004 12:39:02

現在昌時: 9-Aug-2005 20:10:42

フーザー： UEHARA

グループ：TOMOOKA

\# ピーク名 Rt 面積 $[\mu \mathrm{V} . \mathrm{Sec}]$ 面積\%

$\begin{array}{llll}1 & 7.433 & 8172152.343 & 46.41\end{array}$

$2 \quad 8.625 \quad 9435327.250 \quad 53.59$

ピーク総面積 $=17607479.593$ [ $\mu \mathrm{V} . \mathrm{Sec}]$ 


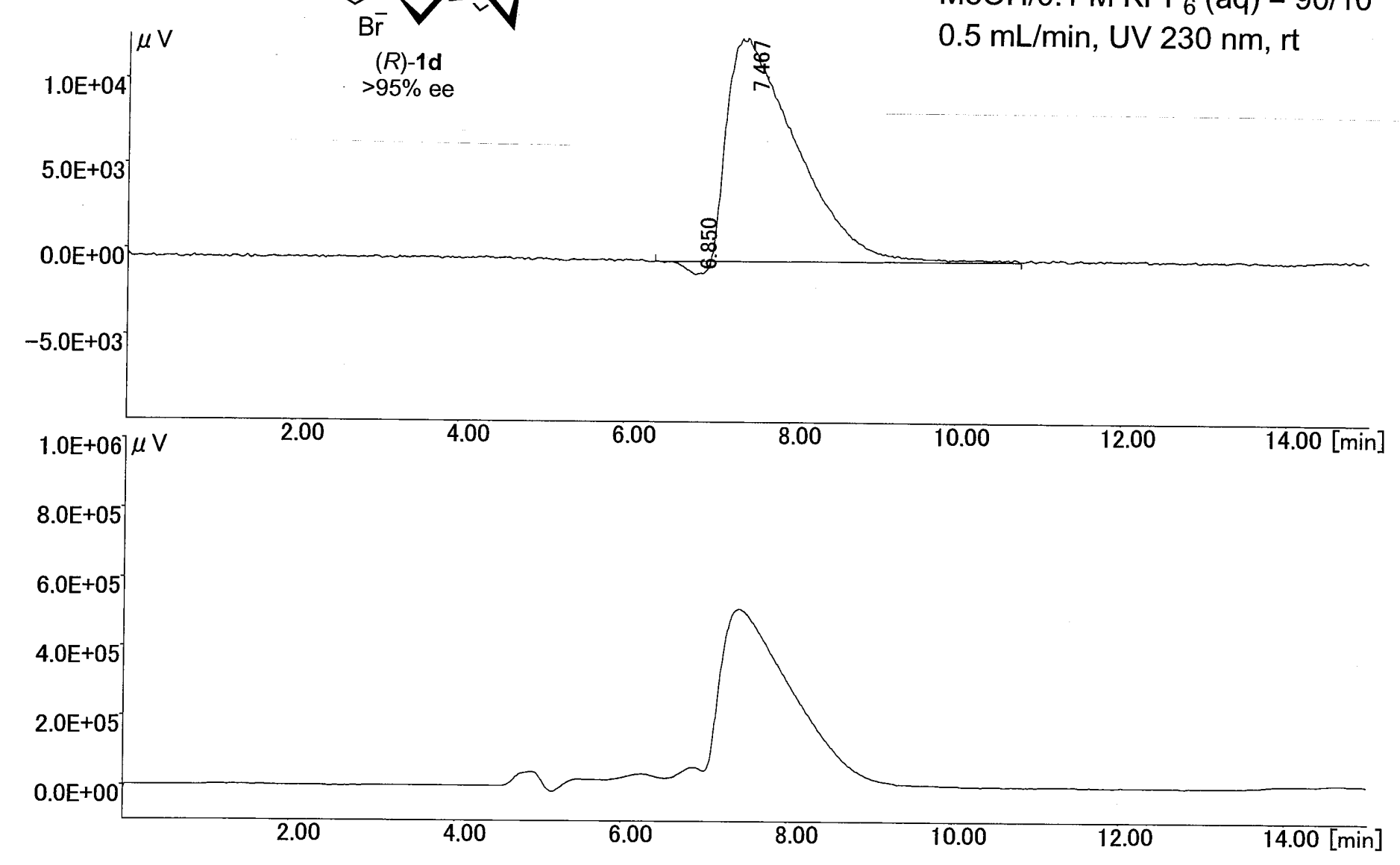

HPLC analysis (CD and UV)

CHIRALCEL OD-RH $(4.6 \times 150 \mathrm{~mm})$

$\mathrm{MeOH} / 0.1 \mathrm{M} \mathrm{KPF}_{6}(\mathrm{aq})=90 / 10$

$0.5 \mathrm{~mL} / \mathrm{min}, \mathrm{UV} 230 \mathrm{~nm}$, rt

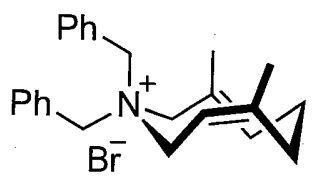

$(R)-1 \mathrm{~d}$

$>95 \%$ ee

ファイル名: KU530-2-001. CH1

コメント:

Vial \# = 1 Rack \#=1

注入日：12-Jun-2004 15:52:52

現在日時： 9-Aug-2005 20:11:36

ユーザー：UEHARA

グループ： TOMOOKA

システムプログラム:MEOH90-KPF10-2-UV230

\# ピーク名 Rt 面積 $[\mu \mathrm{V} . \mathrm{Sec}] \quad$ 面積\%

$\begin{array}{rrrr}1 & 6.850 & 12805.339 & 1.66 \\ 2 & 7.467 & 757400.750 & 98.34\end{array}$

ピーク総面積 $=770206.089 \quad[\mu \mathrm{V} . \mathrm{Sec}]$

ファイル名：KU530-2-001. CH2

コメント:

Vial \# = 1 Rack \#= 1

注入日：12-Jun-2004 15:52:52

現在日時: 9-Aug-2005 20:11:36

ブルザープ： UEHARA

システムプログラム:MEOH90-KPF10-2-UV230

ピークがありません 


\section{CD and UV spectra}

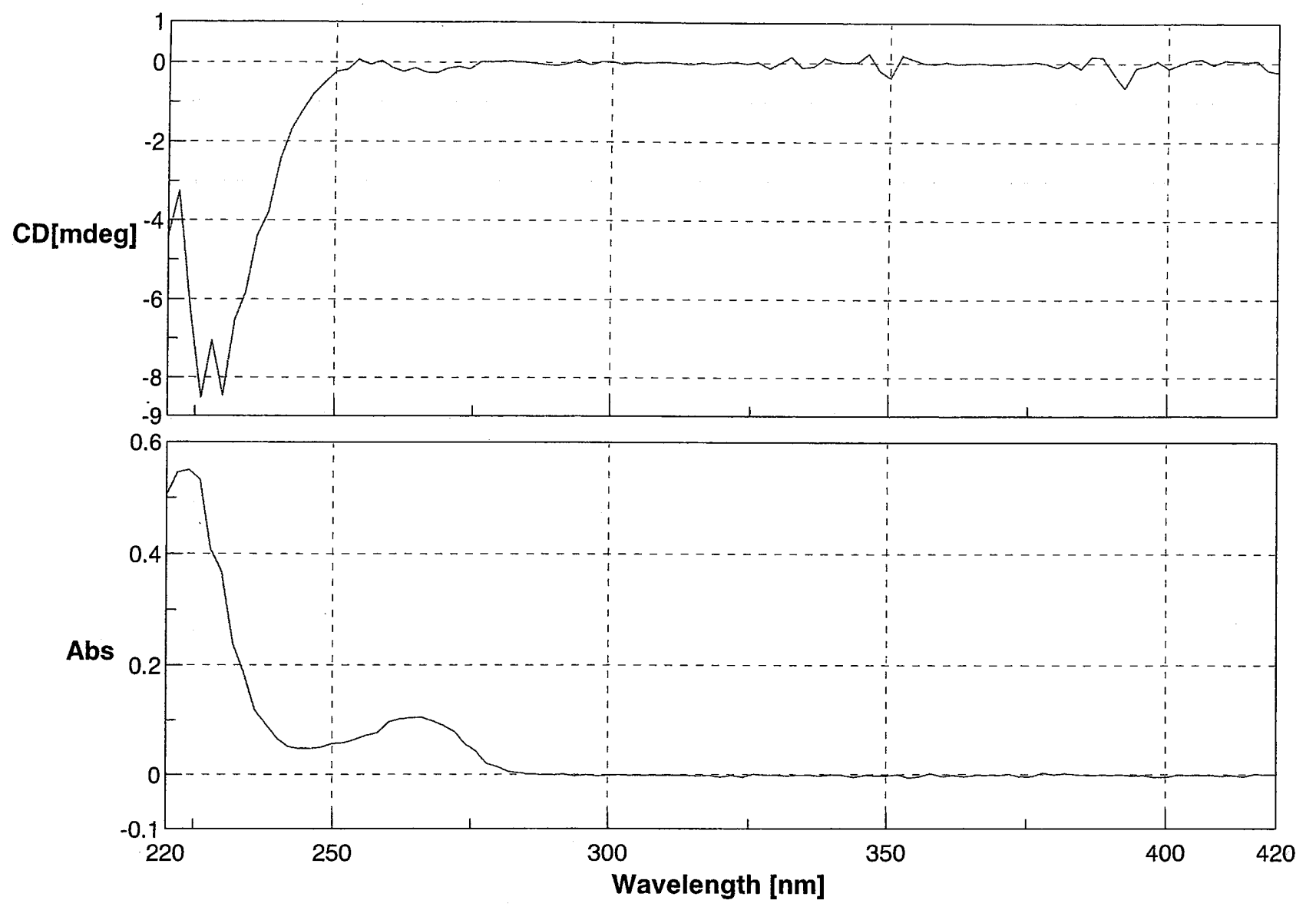

晶時イル名

機種名

シリアル番号

試料名

測定者

犾ント
2004/06/12 15:35

KU518-1. jws

CD-2095

007360875

CD/UV Spectra $t_{R}=7.4$ min for (S)-isomer

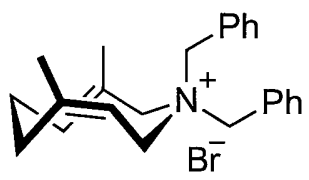

(S)-1d 


\section{CD and UV spectra}
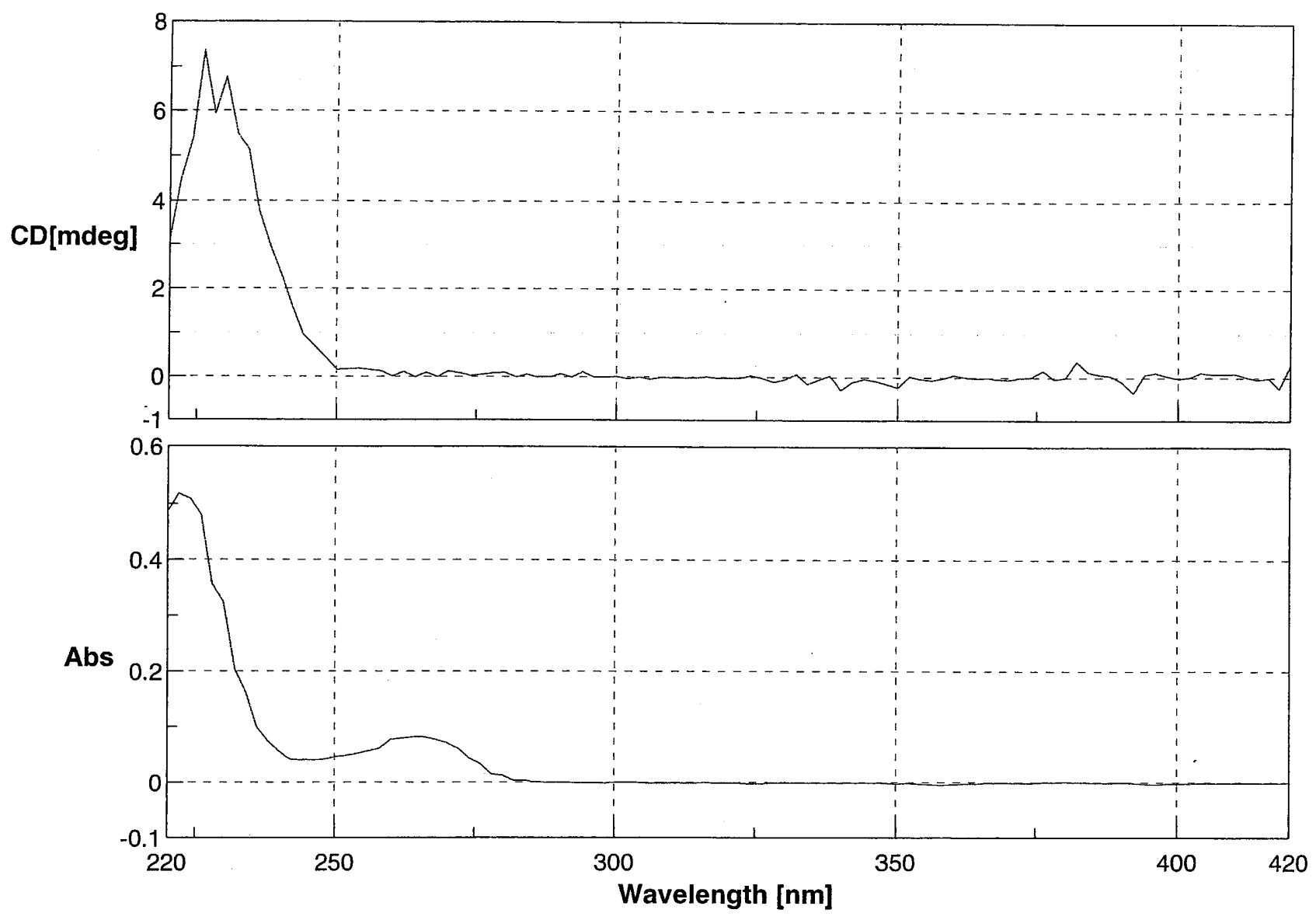

早時 イル名

機喠名

シリアル番号

試料名

測定者

挡ント
2004/06/12 15: 36

KU518-2. jws

CD-2095

007360875

CD/UV Spectra $t_{\mathrm{R}}=8.6 \mathrm{~min}$ for $(R)$-isomer

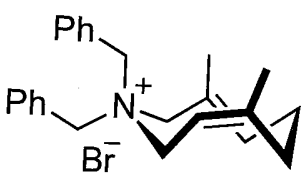

(R)-1d 


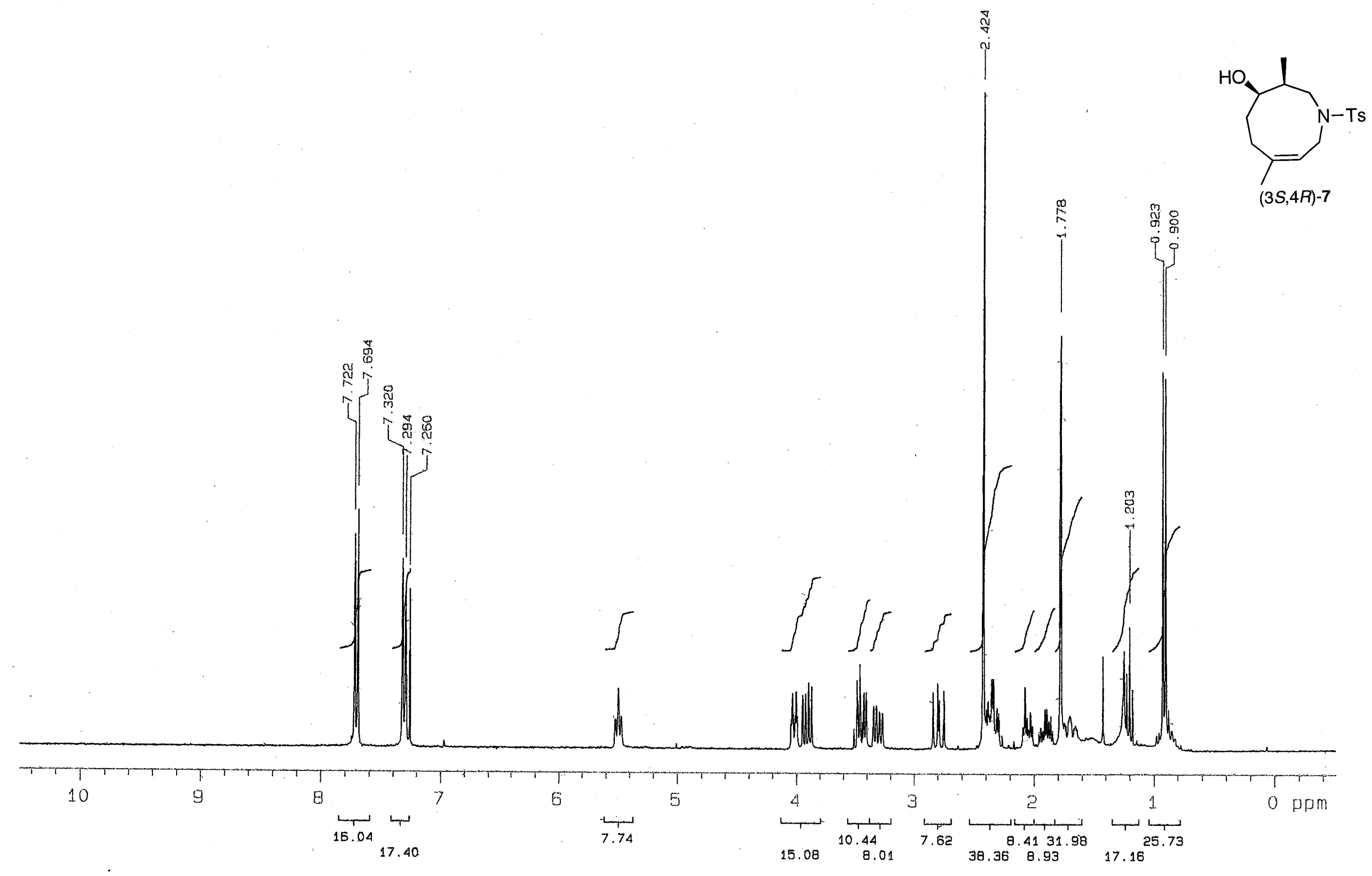




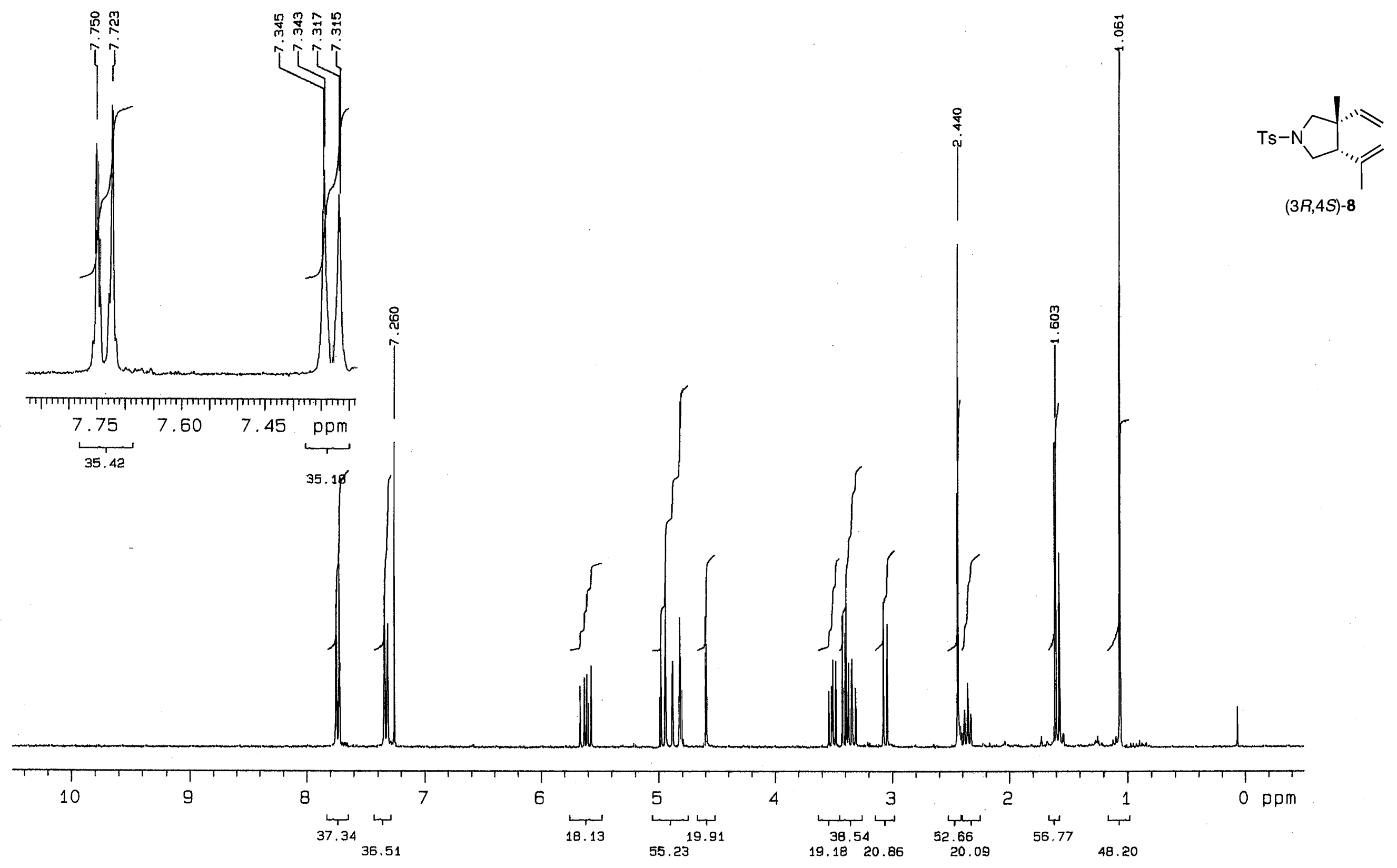



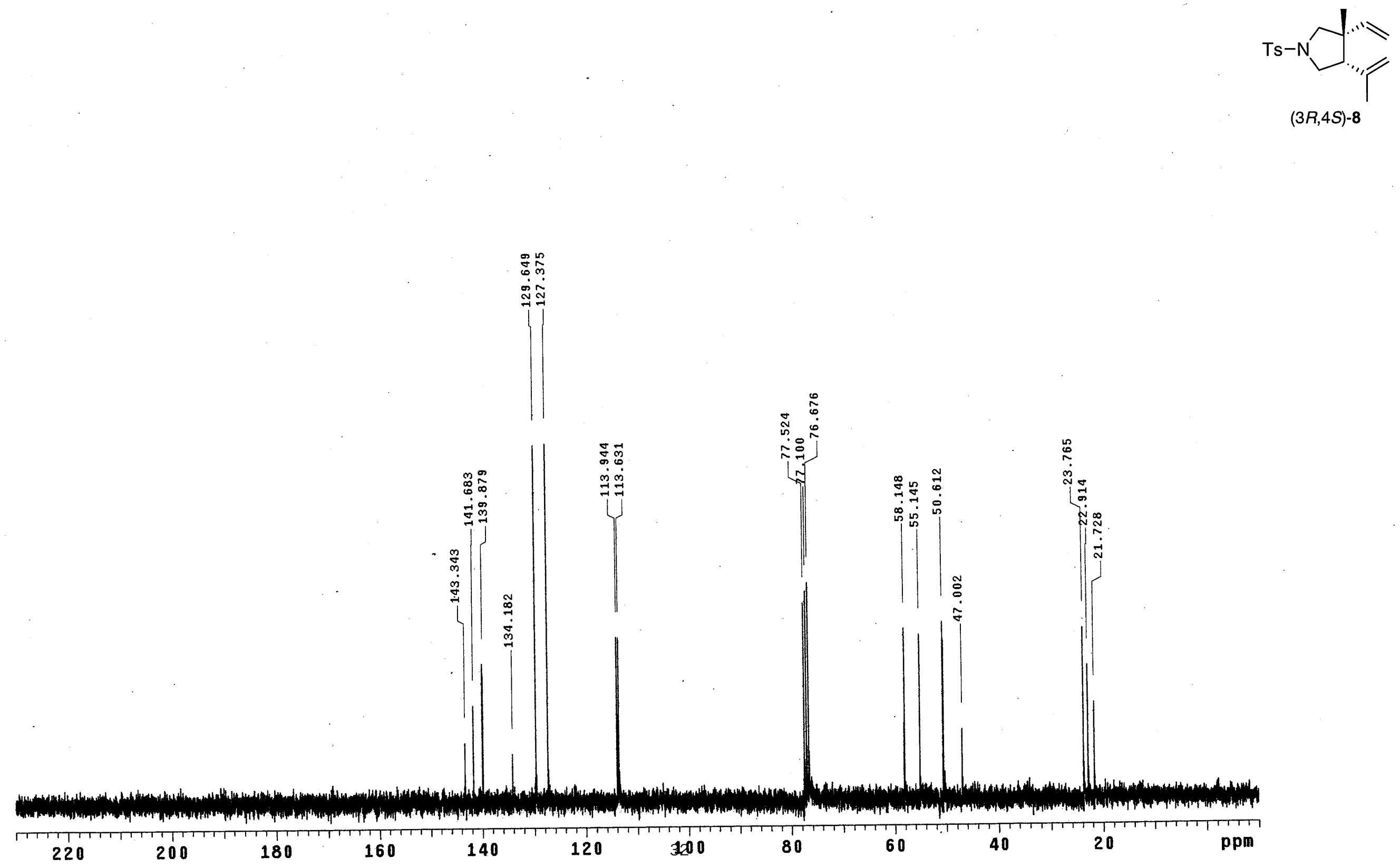